\title{
Substructure Boosts to Dark Matter Annihilation from Sommerfeld Enhancement
}

\author{
Jo Bovy \\ Center for Cosmology and Particle Physics, Department of Physics \\ New York University, New York, NY 10003
}

\begin{abstract}
The recently introduced Sommerfeld enhancement of the dark matter annihilation cross section has important implications for the detection of dark matter annihilation in subhalos in the Galactic halo. In addition to the boost to the dark matter annihilation cross section from the high densities of these subhalos with respect to the main halo, an additional boost caused by the Sommerfeld enhancement results from the fact that they are kinematically colder than the Galactic halo. If we further believe the generic prediction of CDM that in each subhalo there is an abundance of substructure which is approximately self-similar to that of the Galactic halo, then I show that additional boosts coming from the density enhancements of these small substructures and their small velocity dispersions enhance the dark matter annihilation cross section even further. I find that very large boost factors $\left(10^{5}\right.$ to $\left.10^{9}\right)$ are obtained in a large class of models. The implications of these boost factors for the detection of dark matter annihilation from dwarf Spheroidal galaxies in the Galactic halo are such that, generically, they outshine the background gamma-ray flux and are detectable by the Fermi Gamma-ray Space Telescope.
\end{abstract}

\section{INTRODUCTION}

In the standard flat, Gaussian, adiabatic, and scaleinvariant $\Lambda \mathrm{CDM}$ paradigm $\sim 80 \%$ of the matter density of the Universe is in the form of dark matter (DM) [1]. The properties of dark matter are largely unconstrained, although a weakly interacting massive particle (WIMP) with a mass $\sim 100 \mathrm{GeV}-1 \mathrm{TeV}$ has many attractive features, including that it leads to a relic density of dark matter that is remarkably close to the measured value [2]. No interaction of dark matter either with itself or with standard model (SM) particles other than gravity has ever been observed. However, at the present day we cannot exclude that the dark sector is brimming with a rich phenomenology at weak-interaction scales or lower.

One exciting possibility is that dark matter selfannihilates at a level observable in the Galactic halo today. Detecting this self-annihilation seems unlikely considering the standard self-annihilation cross section; $\langle\sigma v\rangle_{\text {ann }} \lesssim 3 \times 10^{-26} \mathrm{~cm}^{3} \mathrm{~s}^{-1}$ for consistency with the observed relic density. However, there are various mechanisms to boost the annihilation signal to a value larger by a few orders of magnitude, e.g., clumpiness of the dark halo $[3,4,5,6]$ or a combination of coannihilations and Sommerfeld enhancement $[7,8]$. The possibility of detecting the DM annihilation signal has attracted much attention recently because of the anomalous excesses of high energy electrons and positrons reported by two different experimental groups: the Payload for Antimatter Exploration and Light-nuclei Astrophysics (PAMELA) satellite, which saw a sharp upturn in the positron fraction $e^{+} /\left(e^{+}+e^{-}\right)$from 10 to 100 $\mathrm{GeV}$ [9], and the Advanced Thin Ionization Calorimeter (ATIC) group, which reported an excess over the expected background in the combined number of electrons and positrons at energies 500 to $800 \mathrm{GeV}$ [10]. The PAMELA result on the positron fraction confirms earlier results $[11,12,13,14,15,16,17]$ and can be explained by $\mathrm{DM}$ annihilation $[18,19,20]$. There is still a good chance that these anomalies can be obviated by conventional astrophysical effects, e.g., pulsars [21, 22, 23, 24]. Nevertheless, a plethora of models has been proposed in the last few months explaining the PAMELA and ATIC results in terms of DM annihilation into electron-positron pairs (e.g., $[25,26,27,28,29,30,31]$ ), a description that also naturally accounts for other experimental anomalies, such as the "WMAP haze" [32, 33, 34], EGRET observations of an excess of high-energy gamma-rays in the Galactic center [35], and recent HESS measurements of the flux of very high-energy electrons [36]. Model building is complicated by the fact that PAMELA did not observe a similar excess in the ratio of anti-protons to protons [37], which means that the coupling of the DM particles to quarks must somehow be suppressed.

The largest obstacle to modeling the PAMELA/ATIC signal with DM annihilation is the very large boost factor $\left(10^{4}\right.$ to $\left.10^{5}\right)$ with respect to the fiducial $\langle\sigma v\rangle_{\text {ann }} \approx$ $3 \times 10^{-26} \mathrm{~cm}^{3} \mathrm{~s}^{-1}$ required to produce an observable annihilation flux. The boost factor from the clumpiness of the dark matter halo is expected to be small $[5,6,38,39]$, typically of the order 1 to 10 . Therefore, a different mechanism must be responsible for the large boosts necessary. One such mechanism that has received much attention lately is the Sommerfeld enhancement $[8,40,41,42,43,44,45,46,47,48]$. The Sommerfeld enhancement is a generic effect present whenever there is an attractive force acting between the dark matter particles, e.g., a Yukawa interaction or a gauge interaction through vector bosons [26]. The main effect of this attractive force is to enhance the annihilation cross section $\sigma v$ with a factor proportional to $\beta^{-1}(\beta \equiv v / c)$; this is known as " $1 / v$ " enhancement. There are regions in the parameter space made up of the dark matter mass $m_{\chi}$, the force carrier mass $m_{\phi}$, and the force "fine-structure constant" $\alpha \equiv$ coupling $^{2} / 4 \pi$ in which the enhancement is near a resonance, where it approximately behaves as $\beta^{-2}$. However, the enhancement saturates at a certain $\beta$ because of the finite range of the attractive force-large 
( $\alpha \sim 10^{-1}$ to $10^{-3}$ ) attractive forces with infinite range lead to a burst of DM annihilation in the first DM halos formed at $z \sim 100-200$ which would lead to observable effects incompatible with measurements of the diffuse extragalactic gamma-ray background today and the cosmic microwave background [49]. Since the typical velocities of dark matter particles in the Galactic halo today are of the order of the velocity dispersion of the halo, which is non-relativistic $\left(\beta \sim 10^{-3}\right)$, the cross section of dark matter particles annihilating in the halo could be significantly enhanced by this Sommerfeld effect.

Both observations $[50,51,52,53,54]$ as well as numerical simulations $[38,55,56,57,58,59,60,61]$ have firmly established that dark matter halos in CDM are not smooth structures, but that they are filled with a large amount of substructure. These substructures are both in the form of subhalos, self-bound objects orbiting in the Galactic halo, and in the form of streams, relics of tidally disrupted subhalos. Since these substructures are overdense regions in the halo, the dark matter annihilation cross section will be correspondingly higher: Annihilation cross sections scale as the density squared. The effects of these kinds of substructure boosts have been studied extensively during the last decade $[5,6,39,62,63,64,65,66,67,68]$. However, these subhalos are not only denser than the Galactic halo in which they orbit, they are also kinematically colder than the Galactic halo, with velocity dispersions that are typically two orders of magnitude smaller [69, 70]. This means that the Sommerfeld enhancement in these subhalos can be many orders of magnitude larger than the Sommerfeld enhancement acting in the Galactic halo taken as a whole.

In addition to this larger boost caused by the small velocity dispersion in the subhalos, these subhalos themselves are filled with an abundance of substructure which is approximately self-similar to that of the main halo. Each of these even smaller substructure has a larger density than the subhalo in which they are orbiting (quantified by the well established concentration-mass relationships for CDM halos [71, 72]) as well as a smaller velocity dispersion. This opens up the possibility that the dark matter annihilation signal in these already cold subhalos can be boosted even further by contribution of their substructure.

In this paper we quantify the magnitude of this new substructure boost and discuss its implications for the detection of dark matter annihilation from subhalos in the Galactic halos. As we will show, the total combined boost from Sommerfeld enhancement and substructure can reach very large values $\left(10^{5}\right.$ to $\left.10^{9}\right)$, and these boosts are only weakly dependent on the assumptions that we have to make about the abundance of substructure and the DM density profile. We use these total boosts together with published DM density profiles of some of the dwarf Spheroidal satellite galaxies of the Milky Way to predict the total flux of gamma-rays from DM annihilation that would be detectable by the Fermi Gamma- ray Space Telescope (Fermi) and find that in most of the models that posit the Sommerfeld enhancement the DM annihilation signal from the dwarf Spheroidals shines above the diffuse extragalactic gamma-ray background, which is the only background signal at the high Galactic latitudes of these satellite galaxies. Finally, we also consider the prospects for detecting smaller subhalos of the Galaxy by computing annihilation fluxes of subhalos in the Via Lactea II simulation. Whether these subhalos will be detectable in the near future depends on the exact details of the Sommerfeld enhancement.

\section{THE SOMMERFELD ENHANCEMENT}

The simplest example of the Sommerfeld effect that contains all of the generic features described in the introduction is that from an attractive Yukawa type force mediated by a spin-0 boson $\phi$ between the DM particles, and we adopt that description here. The Sommerfeld effect is a non-perturbative effect in the sense that in the language of quantum field theory it comes about from Feynman diagrams in which the force carrier is exchanged many times before the annihilation actually happens, giving rise to so called "ladder diagrams". However, we can study the Sommerfeld effect in simple, non-relativistic quantum mechanics (see the appendix in Ref. [26]). We are instructed to solve the radial Schrödinger equation (in natural units)

$$
\frac{1}{m_{\chi}} \frac{\mathrm{d}^{2} \psi(r)}{\mathrm{d} r^{2}}+\frac{\alpha}{r} e^{-m_{\phi} r} \psi(r)=-m_{\chi} \beta^{2} \psi(r),
$$

subject to the boundary condition $\mathrm{d} \psi / \mathrm{d} r=i m_{\chi} \beta \psi$ as $r \rightarrow \infty$. Here $m_{\chi}$ and $m_{\phi}$ are the mass of the dark matter and the force carrier, respectively. Using the substitution $r \rightarrow \alpha m_{\chi} r$ this becomes

$$
\frac{\mathrm{d}^{2} \psi(r)}{\mathrm{d} r^{2}}+\frac{1}{r} e^{-m_{\phi} r /\left(\alpha m_{\chi}\right)} \psi(r)=-\frac{\beta^{2}}{\alpha^{2}} \psi(r),
$$

with boundary condition

$$
\psi \propto e^{i \beta r / \alpha} \quad \text { as } r \rightarrow \infty .
$$

The Sommerfeld enhancement $S$ is then given by

$$
S=\frac{|\psi(\infty)|^{2}}{|\psi(0)|^{2}}
$$

Equation (2) shows that the Sommerfeld enhancement is only a function of two variables, which can be chosen as $\alpha / \beta$ and $\alpha m_{\chi} / m_{\phi}$. The qualitative features of equation (2) have been widely discussed and, therefore, we will merely mention them here without proof (see, e.g., Refs. $[8,26])$. When the kinetic energy of the collision is much larger than the product of the force carrier mass and the coupling, then the Yukawa interaction can be approximated as an attractive Coulomb interaction, for 
which an analytical solution in terms of hypergeometric functions exists. The Sommerfeld enhancement is simply given by

$$
S=\frac{\pi \alpha}{\beta}\left(1-e^{-\pi \alpha / \beta}\right)^{-1} .
$$

From this we see why the Sommerfeld enhancement is often referred to as a " $1 / v$ " enhancement for small velocities $\beta \ll 1$. In the limit of a vanishing interaction $(\alpha / \beta \ll 1)$ the Sommerfeld enhancement disappears as $S \rightarrow 1$. The $1 / v$ behavior, which diverges for small $\beta$ and leads to an overproduction of high-energy photons in the early Universe [49], saturates as a consequence of the finite range of the Yukawa interaction at a velocity $\beta \approx m_{\phi} / m_{\chi}$ and settles on a value $S \approx \alpha m_{\chi} / m_{\phi}$. However, at some parameter combinations the Yukawa potential has bound states, at which there are large boosts to the annihilation cross section which go approximately as $S \propto \beta^{-2}$. These resonance regions, however, are cutoff at small $\beta$ by the finite lifetime of the bound state. We expect these three different behaviors $(1 / v$, saturation at low $v$, and resonance effects from the existence of bound states) to be generic among different models of the Sommerfeld enhancement (see also the discussion in the appendix of Ref. [26]).

Equation (2) can also be numerically integrated efficiently, by integrating the solution $\psi(r)=e^{i \beta r / \alpha}$ inwards from a radius satisfying

$$
\frac{1}{r} e^{-m_{\phi} r /\left(\alpha m_{\chi}\right)} \ll \frac{\beta^{2}}{\alpha^{2}} .
$$

In the Coulomb regime $\left(m_{\chi} \beta^{2} \gg \alpha m_{\phi}\right)$ this means that the initial value for the integration should be such that $r \gg \alpha^{2} / \beta^{2}$; otherwise we should start at $r \gg \alpha m_{\chi} / m_{\phi}$. Figure 1 shows the Sommerfeld enhancement in the parameter plane made of $\alpha$ and $m_{\phi} / m_{\chi}$. All of the qualitative features we discussed above are present: In the Coulomb regime (lower part of the two panels) we see that the enhancement scales as $\alpha$, saturating at $S \approx 1$ when $\alpha$ becomes small; The fact that the upper left corner of both panels is approximately the same shows that the Sommerfeld enhancement saturates at $\beta \approx m_{\phi} / m_{\chi}$; The sharp diagonal lines in the upper right of both panels show the resonance region, in which the enhancement is sharply peaked. Because of the scaling properties of equation (2), shifting $\log \beta$ is equivalent to shifting both $\log \alpha$ and $\log m_{\phi} / m_{\chi}$ by the same amount. Therefore, the $\beta$ behavior of the enhancement can also be read off from these figures along diagonals. Inspection of the resonance diagonals in the second panel of Figure 1 shows that the enhancement grows as $\beta^{-2}$ in these regions.

Of course, when considering the Sommerfeld enhancement of a halo of a certain velocity dispersion $\sigma_{v}$ we need to average the Sommerfeld enhancement over the distribution of relative velocities of that halo. We will approximate the one-particle velocity distribution of the halo as a single truncated Maxwell-Boltzmann distribution with
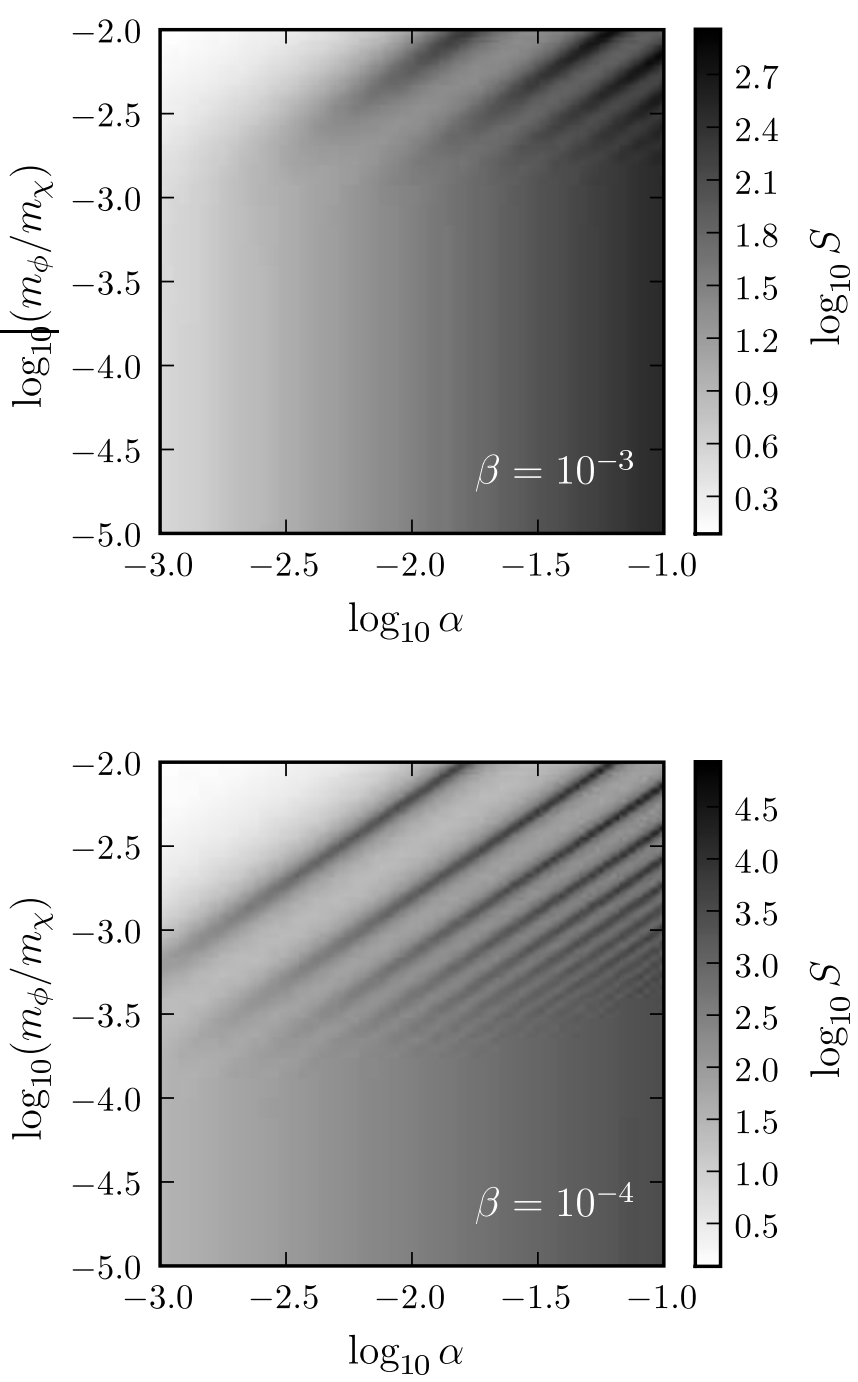

FIG. 1: Sommerfeld enhancement as a function of the particle physics parameters for two different values of the relative velocity $\beta$. Because of the scaling properties of equation (2) the behavior of the Sommerfeld enhancement as a function of $\log _{10} \beta$ can actually be read off along diagonals in the plane.

velocity dispersion $\sigma_{v}$

$$
f(v) \propto\left\{\begin{array}{rl}
v^{2} e^{-v^{2} / 2 \sigma_{v}^{2}} & v \leq v_{\mathrm{esc}} \\
0 & v>v_{\mathrm{esc}}
\end{array}\right.
$$

This approximation of a single Maxwell-Boltzmann distribution for the velocity distribution means that we assume that the halo has a constant velocity dispersion over the halo, i.e., that the halo is isothermal. This is not necessarily the case - indeed, it is not if we believe that the density profiles of DM halos are well fit by Einasto or NFW profiles - but it is not a bad assumption for intermediate distances from the halo's center, i.e., between the innermost and outermost parts of the halo [73]. A more detailed treatment of the Sommerfeld enhancement 
in subhalos should take the non-uniform velocity dispersion in subhalos into account. Note that in the pure $1 / v$ regime the averaging will tend to sustain the $1 / v$ behavior up to a factor $\mathcal{O}(1)$, as we have that

$$
\int_{0}^{\infty} \mathrm{d} v v^{2} e^{-v^{2} / 4 \sigma_{v}^{2}} S(v) / \int_{0}^{\infty} \mathrm{d} v v^{2} e^{-v^{2} / 4 \sigma_{v}^{2}} \propto \frac{1}{\sigma_{v}}
$$

since the truncation does not significantly influence the result of these integrals. Similarly, in the resonance region the $1 / v^{2}$ behavior is conserved by the averaging. When the enhancement has leveled off the averaging has no effect.

In Figure 2 we show this averaged Sommerfeld enhancement for four different decades of velocity dispersion. The overall behavior of the Sommerfeld enhancement seen in Figure 1 is indeed conserved well by the averaging: this has to do with the fact that the most probable relative velocity in a Maxwell-Boltzmann relative velocity distribution is the velocity dispersion. The Sommerfeld enhancement attains very large values for subhalos with very small velocity dispersions, mostly, but not exclusively, in the resonance regions. This means that the smallest subhalos that exist in CDM will get large boosts to their DM annihilation from Sommerfeld enhancement.

\section{SUBSTRUCTURE BOOSTS FROM SOMMERFELD ENHANCEMENT}

The photon flux from DM annihilation from a solid angle $\Delta \Omega$ along a given line-of-sight (los) is given by

$$
\begin{aligned}
\frac{\mathrm{d} N_{\gamma}}{\mathrm{d} A \mathrm{~d} t}= & \int_{E_{\mathrm{th}}}^{m_{\chi}} \mathrm{d} E \sum_{i} \frac{\mathrm{d} N_{\gamma, i}}{\mathrm{~d} E} \frac{\langle\sigma v\rangle_{i}}{m_{\chi}^{2}} S\left(m_{\phi} / m_{\chi}, \alpha, \sigma_{v}\right) \\
& \times \int_{\Delta \Omega} \frac{\mathrm{d} \Omega}{4 \pi} \int_{\operatorname{los}} \mathrm{d} l \rho^{2}(l),
\end{aligned}
$$

where $E_{\mathrm{th}}$ is the threshold energy of the detector, $i$ denotes (possibly) different final states of the DM annihilation, $\frac{\mathrm{d} N_{\gamma, i}}{\mathrm{~d} E}$ is the spectrum of the annihilation to that state, $\langle\sigma v\rangle_{i}$ is the annihilation cross section to state $i$ (without the Sommerfeld enhancement), $S\left(m_{\phi} / m_{\chi}, \alpha, \beta\right)$ is the Sommerfeld enhancement which we have factored out of the cross section, and $\rho(l)$ is the DM density along the line-of-sight. We emphasize that this framework also allows us to consider different final annihilation products, such as the electrons and positrons observed by PAMELA/ATIC.

We will focus our analysis on the "structure quantity" $\mathcal{L}(M)$, which we define here as

$$
\mathcal{L}(M) \equiv S\left(m_{\phi} / m_{\chi}, \alpha, \sigma_{v}\right) \int_{\Delta \Omega} \frac{\mathrm{d} \Omega}{4 \pi} \int_{\operatorname{los}} \mathrm{d} l \rho^{2}(l)
$$

This structure quantity does not only depend on the mass and internal properties of the DM halos in which we are interested, it depends on the particle physics parameters as well. We will keep all of the other factors in equation (9) fixed. However, in order to derive actual numbers for the photon flux from DM annihilation, we briefly describe the other quantities appearing in equation (9).

\section{A. Some details of the particle physics model}

For the annihilation cross section prior to any Sommerfeld enhancement we will adopt the fiducial value $\langle\sigma v\rangle=3 \times 10^{-26} \mathrm{~cm}^{3} \mathrm{~s}^{-1}$, and we choose a fiducial mass for the DM particle of $m_{\chi}=700 \mathrm{GeV}$. The energy spectrum of the DM annihilation depends on the model used to describe the DM and its interactions; it is outside of the scope of this paper to provide a detailed treatment of this for different DM models. Therefore, we will adopt an annihilation spectrum for DM annihilation into photons appropriate for the newer models that were proposed to explain the PAMELA/ATIC results. Specifically, we use a gamma ray spectrum from a cascade annihilation model. In these models the DM annihilates into a new light degree of freedom which then in turn decays into leptons and photons. The injection spectrum per DM annihilation is given by [74]

$$
\begin{aligned}
\frac{\mathrm{d} N_{\gamma}}{\mathrm{d} E}= & \frac{\alpha_{\mathrm{EM}}}{\pi} \frac{1+\left(1-E / m_{\chi}\right)^{2}}{E} \\
& \times\left\{-1+\ln \left(4\left(1-E / m_{\chi}\right)\right)-2 \ln \left(\frac{m_{l}}{m_{\chi}}\right)\right\},
\end{aligned}
$$

in which $\alpha_{\mathrm{EM}}$ is the electromagnetic coupling constant and $m_{l}$ is the mass of the leptons created in the process $\chi \chi \rightarrow l^{+} l^{-} \gamma$. Integrating this spectrum using an energy range appropriate for Fermi we find that

$$
\int_{4 \mathrm{GeV}}^{250 \mathrm{GeV}} \mathrm{d} E \frac{\mathrm{d} N_{\gamma}}{\mathrm{d} E} \approx 0.5
$$

We find a similar number if we integrate the spectrum from one cascade step. It is interesting to note that these photon spectra have fewer photons in them, about an order of magnitude less, than the annihilation spectra from neutralino annihilation which are often used $[75,76]$. Of course, since the energy spectrum contributes a multiplicative factor to the DM annihilation flux, the effect of different annihilation spectra can be incorporated by simply multiplying our results with the appropriate factor.

\section{B. The Dark Matter profile}

Since the photon flux from DM annihilations depends on the density squared of the DM the exact form of the inner density profile has important ramifications for the detectability of DM annihilation. The density profile of 

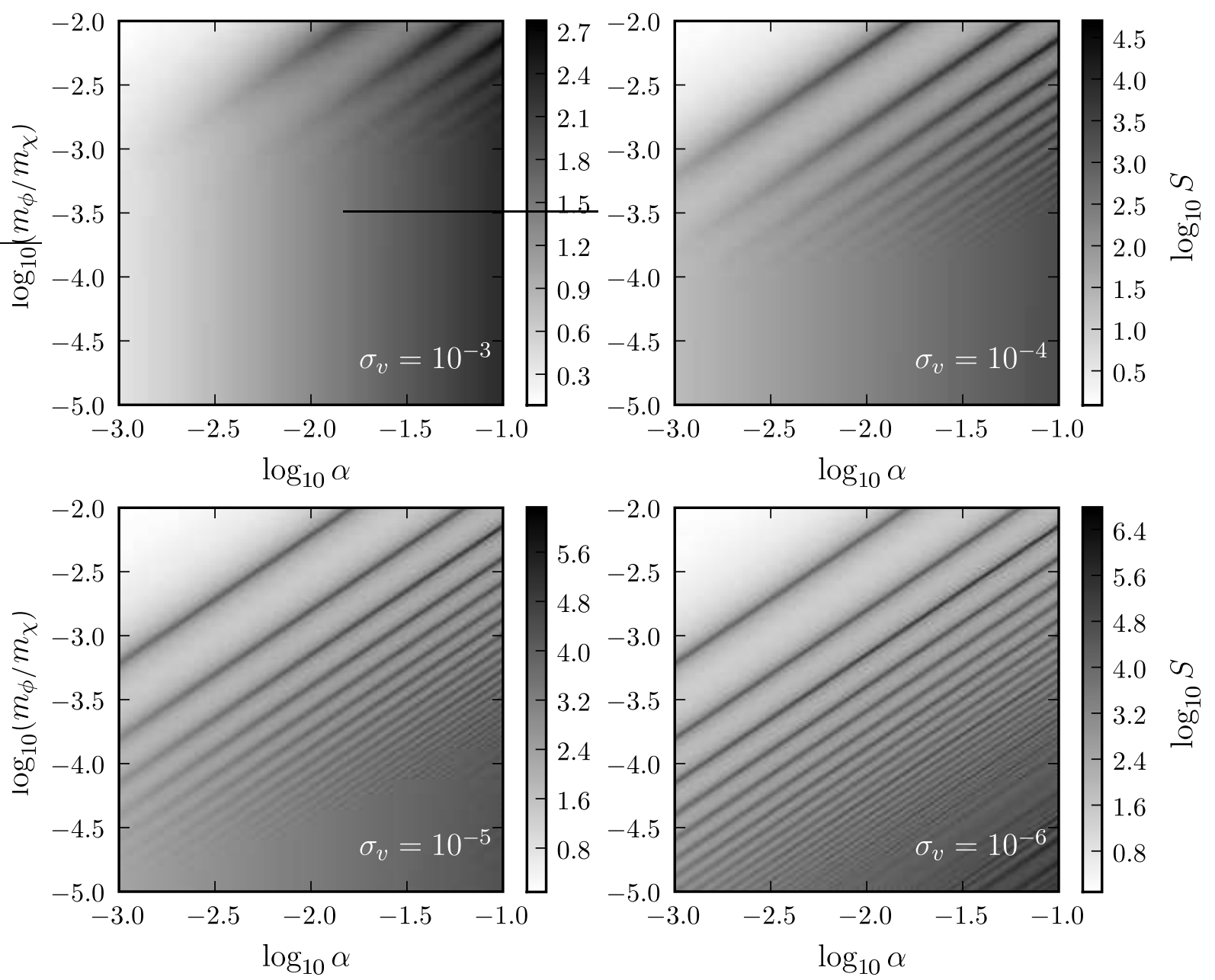

FIG. 2: Sommerfeld enhancement factor as a function of the particle physics parameters assuming a one-particle MaxwellBoltzmann velocity distribution with velocity dispersion $\sigma_{v}$ for four different orders of magnitude of the velocity dispersion (all velocities are relative to the speed of light).

dark matter halos has been discussed extensively in the last few years. While the shape of the outer density profile seems to have converged in the various numerical simulations of DM halos and a consensus has been reached, the detailed shape of the inner density profile is still a matter of debate. The main point of contention is whether the density profile is cusped near the center of the DM halo, and if so, how steep this cusp is, or whether the density asymptotes to a constant value at the center. Since this discussion has a non-negligible impact on the prospects for DM annihilation detection, we will briefly discuss the various plausible density profiles.

The inner structure of DM halos is one of the key predictions of the CDM paradigm, and over a decade ago it was shown that the spherically averaged density profiles of DM halos are well described by a universal profile, the so-called NFW profile [77, 78, 79], depending only on the virial mass of the halo and its concentration. Moreover, the concentration was shown to depend systematically on the halo mass $[71,72,78,79,80,81]$. This profile was found to provide a good fit to many numerical simulations (e.g., [82, 83, 84]). While there seems to be a consensus between different numerical simulations on the shape of the outer density profile, no agreement was reached on the inner density structure of the DM halos found in the simulations. The NFW profile has an inner slope of -1, other studies, however, found evidence for steeper inner slopes [85, 86, 87, 88, 89, 90, 91], shallower inner slopes [92], or didn't find any convergence toward a power-law behavior [93]. Even the universality of the density profile has been brought into question [94, 95].

More recently, high-resolution numerical simulations have essentially ruled out the steepest cusps, such as Moore's profile, which has an inner cusp a with logarithmic slope of -1.5 , however steeper inner cusps than the original NFW profile are still observed in numerical simulations [96]. Other recent simulations find inner slopes that are shallower than an NFW profile $[97,98,99]$. It also has become apparent in recent years that the inner density profile of DM halos might not be described by a 
power-law divergence, but that, on the contrary, the logarithmic slope becomes ever shallower, leading to a cored density profile $[61,98,99,100,101,102,103,104]$. Therefore, density profiles such as the Einasto profile [105, 106] have been shown to provide an excellent fit to the numerical simulations with the highest resolution yet $[61,98]$.

The implications of the exact form of the inner density profile have been widely discussed before in the context of various DM annihilation models (e.g., [75, 107, 108, $109,110,111,112,113,114,115,116])$. Roughly speaking, the annihilation flux is smaller for inner density profiles which are cored, and larger for inner density profiles which show a cuspy behavior. The steeper the slope of the inner cusp, the larger the DM annihilation flux (for obvious reasons). Since the core vs. cusp debate is still undecided we will consider DM distribution models of both types.

The cuspy DM profile we will use is a generalized NFW profile $(\mathrm{GNFW})$ :

$$
\rho(r)=\frac{\rho_{s}}{\tilde{r}^{\gamma}(1+\tilde{r})^{3-\gamma}} ; \quad \tilde{r}=r / r_{s},
$$

in which $\rho_{s}$ is a characteristic density and $r_{s}$ a characteristic (scale-)radius. The original NFW profile is recovered for $\gamma=1$ [79], which we will use as the fiducial model in what follows. Varying the value of the inner logarithmic slope $\gamma$ will allow us to describe the effect of steeper and shallower inner slopes than the NFW model. Note that this functional form does not include the exact form of Moore's profile; this need not concern us since inner cusps as steep as Moore's profile are excluded by the latest numerical simulations.

The contribution of the DM density profile on the structure quantity $\mathcal{L}$ can be simply evaluated in the context of any density model. As in Ref. [65] we assume that the distance to the subhalo $D$ is much larger than the scale-radius of the DM halo. For an NFW profile $90 \%$ of the flux originates from the region within one scale-radius, and we will, conservatively, only take contributions to the DM annihilation flux coming from this region for all of the different profiles. Therefore, we find that

$$
\int_{\Delta \Omega} \frac{\mathrm{d} \Omega}{4 \pi} \int_{\operatorname{los}} \mathrm{d} l \rho^{2}(l)=\mathcal{N}_{1}(\gamma) \frac{\rho_{s}^{2} r_{s}^{3}}{D^{2}}
$$

The normalization of this relationship depends on the logarithmic slope of the inner cusp and is given by

$$
\mathcal{N}_{1}(x)=4 \pi \frac{2^{2 x-5}\left(-16+11 x-2 x^{2}\right)}{-30+47 x-24 x^{2}+4 x^{3}} .
$$

The normalization for the NFW profile is $7 \pi / 6$ and it depends strongly on the value of the logarithmic slope of the inner density profile, as can be seen in Figure 3. The bottom panel of Fig. 3 shows the fraction of the DM annihilation flux coming from region within one scaleradius of the halo. For reasonable values of the inner slope this fraction is in the range 80 to $95 \%$.

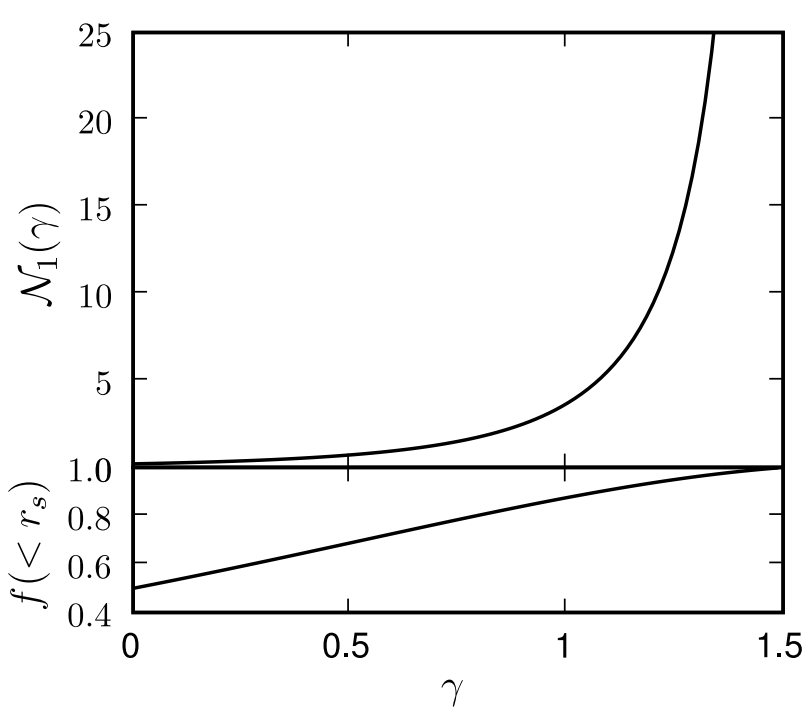

FIG. 3: Top: Normalization of the $\mathcal{L} \propto \rho_{s}^{2} r_{s}^{3} / D^{2}$ relation of equation (14) for the GNFW profile. The functional dependence of the normalization on the exponent $\gamma$ in the profile given in equation (13) is given in equation (15). Bottom: Fraction of the emission that comes from the region within $r_{s}$.

The Einasto profile is characterized by an everdecreasing logarithmic inner slope. It's functional dependence on the radius is given by

$$
\rho(r)=\rho_{-2} \exp \left[-\frac{2}{\alpha}\left\{\left(\frac{r}{r_{-2}}\right)^{\alpha}-1\right\}\right] .
$$

Here, $\rho_{-2}$ is the density at the radius $r_{-2}$ where the local slope is -2 and $\alpha$ is a shape parameter often fixed at a value $\sim 0.17$ [61]. As above, assuming $D \gg r_{-2}$, calculating the flux originating from within the radius $r_{-2}$, we find that

$$
\int_{\Delta \Omega} \frac{\mathrm{d} \Omega}{4 \pi} \int_{\operatorname{los}} \mathrm{d} l \rho^{2}(l)=\mathcal{N}_{2}(\alpha) \frac{\rho_{-2}^{2} r_{-2}^{3}}{D^{2}} .
$$

The normalization depends on the value of $\alpha$ and is given by

$$
\mathcal{N}_{2}(x)=4 \pi 2^{-6 / \alpha} e^{4 / \alpha} \alpha^{-1+3 / \alpha} \gamma\left[\frac{3}{\alpha}, \frac{4}{\alpha}\right],
$$

where $\gamma[a, z]$ is the lower incomplete gamma function. The dependence of this normalization on $\alpha$ is shown in Figure 4. In order to compare the Einasto profile as described here to the GNFW profile, we can relate the parameters $\left(\rho_{s}, r_{s}\right)$ of the GNFW profile to the parameters $\left(\rho_{-2}, r_{-2}\right)$ of the Einasto model by calculating the radius at which the logarithmic slope of the density versus radius profile equals -2 . Thus, we find that

$$
r_{-2}=r_{s}, \quad \rho_{-2}=\frac{\rho_{s}}{2^{3-\gamma}} .
$$




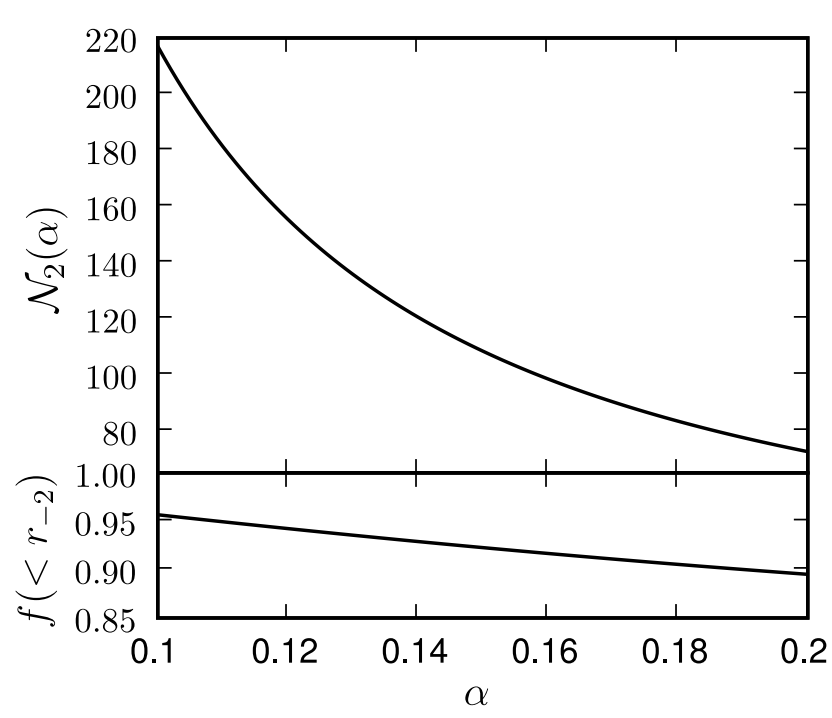

FIG. 4: Top: Normalization of the $\mathcal{L} \propto \rho_{-2}^{2} r_{-2}^{3} / D^{2}$ relation of equation (17) for the Einasto profile. The functional dependence of the normalization on $\alpha$ in the profile given in equation (16) is given in equation (18). Bottom: Fraction of the emission that comes from the region within $r_{-2}$.

Note that the DM annihilation flux from the Einasto profile, although it seems larger from Figs. 3 and 4 , is actually smaller in general, since there is a factor of $2^{6-2 \gamma}$ involved in going from one to the other.

Further on it will sometimes be useful to describe the DM density profiles by different parameters than a characteristic density and radius. Another set of parameters is given by a concentration parameter and a mass. The virial radius $r_{\text {vir }}$ of a halo is defined to be the radius within which the mean density of the halo is $\Delta$ times the critical density $\rho_{\mathrm{c}}$, where $\Delta=178 \Omega_{m}^{0.45} \approx 100[117,118]$. The virial mass is simply the mass contained within a sphere of radius $r_{\text {vir }}$

$$
M_{\mathrm{vir}} \equiv \frac{4 \pi}{3} \Delta \rho_{\mathrm{c}} r_{\mathrm{vir}}^{3}
$$

The concentration for both the GNFW profile as for the Einasto profile is then defined by

$$
c \equiv \frac{r_{\text {vir }}}{r_{-2}}
$$

A characteristic mass of a halo is given by the virial mass defined above. The virial mass of the GNFW profile in terms of the characteristic density $\rho_{s}$ and the characteristic radius $r_{s}$ is

$$
M_{\mathrm{vir}}=4 \pi \rho_{s} r_{s}^{3} f(c),
$$

in which the function $f(c)$ is given by

$$
f(c)=\frac{c^{3-\gamma}}{3-\gamma}{ }_{2} F_{1}(3-\gamma, 3-\gamma ; 4-\gamma ;-c) \text {. }
$$

The function ${ }_{2} F_{1}(a, b ; c ; z)$ is the Gauss hypergeometric function. In the $\gamma=1$ case $f(c)$ is given by the familiar form $f(c)=\ln (1+c)-c /(1+c)$. The virial mass for the Einasto profile in terms of the characteristic density $\rho_{-2}$ and the characteristic radius $r_{-2}$ is

$M_{\mathrm{vir}}=4 \pi \rho_{-2} r_{-2}^{3} \frac{1}{\alpha} \exp \left(\frac{3 \ln \alpha+2-\ln 8}{\alpha}\right) \gamma\left[\frac{3}{\alpha}, \frac{2}{\alpha} c^{\alpha}\right]$.

In this last expression $\gamma[a, z]$ is the lower incomplete gamma function. The density can then be expressed in terms of the concentration by using the definition of the virial mass given in equation (20).

Another pair of parameters that one can use to characterize the DM density profile is the maximum circular velocity $v_{\max }$ and the radius at which this maximum occurs, $r_{\max }$. These parameters are especially well-suited when dealing with numerical simulations, since they can be readily read off from the raw simulation data. The circular velocity is given by

$$
v_{\mathrm{c}}^{2}(r) \equiv \frac{G M(r)}{r}=v_{\mathrm{c}}^{2}\left(r_{\mathrm{vir}}\right) \frac{c}{f(c)} \frac{f(x)}{x}
$$

in which $x \equiv r / r_{-2}$ and $f(x)$ is defined in equation (23) in the case of a GNFW profile and is given by $\gamma\left[\frac{3}{\alpha}, \frac{2}{\alpha} x^{\alpha}\right]$ in the case of an Einasto profile. One can find $r_{\max }$ by (numerically) solving the equation

$$
\frac{\mathrm{d}}{\mathrm{d} x}\left[\frac{f(x)}{x}\right]=0
$$

and $v_{\max }$ follows then immediately.

\section{Calculating the total boost}

As is customary, we will write the total structure quantity $\mathcal{L}(M)$ as a sum of a smooth component and a substructure component:

$$
\mathcal{L}(M)=\mathcal{L}_{\mathrm{sm}}(M)+\mathcal{L}_{\mathrm{sub}}(M) .
$$

The smooth contribution to the structure quantity is the product of the Sommerfeld enhancement $S$ and the lineof-sight integral over the DM density squared, given for the different DM density profiles in equations (14) and (17). Therefore,

$$
\mathcal{L}_{\mathrm{sm}}(M)=S \mathcal{L}_{\mathrm{sm}, v=c}(M)
$$

in which $\mathcal{L}_{\mathrm{sm}, v=c}$ is the luminosity that would come from the smooth component of the halo in the absence of Sommerfeld enhancement.

We can now write

$$
\begin{aligned}
\mathcal{L}(M) & =S \mathcal{L}_{\mathrm{sm}, v=c}(M)+\mathcal{L}_{\mathrm{sub}}(M) \\
& =\left[S+\frac{\mathcal{L}_{\mathrm{sub}}(M)}{\mathcal{L}_{\mathrm{sm}, v=c}(M)}\right] \mathcal{L}_{\mathrm{sm}, v=c}(M) .
\end{aligned}
$$


We will from now on write the substructure boost as $B(\mathrm{M})$. Then we have the following

$$
\begin{aligned}
B(M) & \equiv \frac{\mathcal{L}_{\mathrm{sub}}(M)}{\mathcal{L}_{\mathrm{sm}, v=c}(M)}=\frac{1}{\mathcal{L}_{\mathrm{sm}, v=c}(M)} \int \mathrm{d} m \frac{\mathrm{d} N}{\mathrm{~d} m} \mathcal{L}(m) \\
& =\frac{1}{\mathcal{L}_{\mathrm{sm}, v=c}} \int \mathrm{d} m \frac{\mathrm{d} N}{\mathrm{~d} m}[S(m)+B(m)] \mathcal{L}_{\mathrm{sm}, v=c}(m) .
\end{aligned}
$$

Here we have introduced the subhalo mass function $\mathrm{d} N / \mathrm{d} m$, which gives the abundance of subhalos of mass $m$.

Before proceeding we should specify the upper and lower limits of the integral appearing in equation (30). The integration over ever smaller substructures is cutoff at the size of the smallest subhalos. The mass scale at which this cut-off occurs is the thermal freestreaming scale, a DM model-dependent quantity, which in many DM models lies in the range $10^{-6}$ to $10^{-12} M_{\odot}$ $[119,120,121,122,123]$. The upper limit to this integral is some fraction $q$ of the halo mass $M$, since the abundance of substructure does not continue up to this mass scale. In the following we will set $q$ equal to 0.1 .

In order to calculate the total boost from substructure to the annihilation cross section, we will write all of the quantities appearing in equation (30) as a function of the mass $m$. For instance, from numerical simulations, the subhalo mass function is well constrained to follow a power-law type behavior:

$$
\frac{\mathrm{d} N}{\mathrm{~d} \ln m} \propto\left(\frac{m}{M}\right)^{n} .
$$

The value of the exponent $n$ is still under debate. The two highest resolution $N$-body simulations yet, the Aquarius and Via Lactea projects, both find values for $n$ that put roughly equal amounts of mass per subhalo mass decade. While the Via Lactea simulation seems to prefer a value $n=-1[6,124]$, which would lead to a logarithmically diverging total mass in substructures if the subhalo abundance were not cut off at the thermal freestreaming limit of the dark matter, the Aquarius project prefers a value $n=-0.9$ [61]. As the Aquarius project has made detailed convergence studies that support their claim for a value of $n$ that is shallower than -1 , we will adopt the value $n=-0.9$ as our fiducial value, although even shallower slopes have also been observed [125]. The same slope has also been observed in other simulations [126].

With the exponent $n$ fixed, we turn to the normalization of the relation given in equation (31). Again, different high-resolution numerical simulations give different answers to this question as it relates to the abundance of subhalos of Galactic subhalos. The problem of the normalization is complicated by the fact that numerical simulations have only recently begun to be able to resolve substructure in subhalos in a Galaxy-size halo. While the simulations generally agree that the resolved substructure abundance of subhalos in the host halo adds up to $\sim 10 \%$ of the total mass of the halo $[55,58,64,91,127,128]$, the question of whether the substructure abundance in subhalos is just a scaled-down version of the substructure abundance in the host halo remains unresolved. One would not expect the abundance to be self-similar, since, while substructure in both the main host halo and subhalos gets diminished by tidal disruptions, substructure in the subhalos does not get replenished by the infall of halos from the field, as is the case for the host halo. Nevertheless, many numerical simulations do find approximately self-similar substructure abundances [57, 129]. Recently, the Aquarius simulation has concluded from its simulation that the substructure abundance is not self-similar, although the deviation from the self-similar relation is small [61]. Therefore, we will adopt a normalization here which places $10 \%$ of the mass of the subhalo in the form of "resolved" substructure, where we adopt the definition that resolved substructure is in halos of mass $\gtrsim 10^{-5} \mathrm{M}$.

In the previous section we calculated $\mathcal{L}_{\mathrm{sm}, v=c}$ for the different DM density profiles and found in all cases that

$$
\mathcal{L}_{\mathrm{sm}, v=c}(m) \equiv \mathcal{L}_{\mathrm{sm}, v=c}(\rho(m), r(m)),
$$

for a characteristic density $\rho$ and radius $r$, the exact definition of which depends on the specific DM density profile used. In order to find the mass dependence of $\mathcal{L}_{\mathrm{sm}, v=c}$, we use the description of the DM density profile in terms of virial mass $M_{\text {vir }}$ and concentration $c$. We then make use of the fact that many simulations have found a relation between the concentration of halos and their mass $[71,72,80,81]$, such that everything depends only on the mass. This relation is well established over a large range in halo masses, and we will adopt the particular relationship found in Ref. [130]

$$
c=10.5\left(\frac{M_{\mathrm{vir}}}{10^{12} M_{\odot}}\right)^{-0.11},
$$

in good agreement with the results of other, similar analyses [71, 131], although the normalization of the concentration-mass relation is about $15 \%$ lower than the original relation. However, the concentration of subhalos is generally larger than the concentration of field halos of the same mass as a result of tidal mass-loss $[55,132,133,134,135]$. This is a small effect, of order $10 \%$, and it does not play a large role in the results we obtain below. This concentration-halo mass concentration has not just been established in numerical simulations: statistical weak lensing analysis of luminous red galaxies and clusters from the Sloan Digital Sky Survey (SDSS) has shown that the halo profiles of these objects are consistent with the NFW profile and mass-concentration relation given above [136].

The only remaining quantity in equation (30) that we haven't related to the mass of the subhalo yet, is the Sommerfeld enhancement factor $S$. The Sommerfeld enhancement depends on the mass of the subhalo through the velocity dispersion. Therefore, we need to relate the 
velocity dispersion of a DM subhalo to its mass. It is important to note that all we really need is an approximate relationship between the velocity dispersion and the halo mass which fits well the range of Galactic size halos to a scale not much smaller than the mass scale of the smallest dwarf Spheroidals known today, since in most cases the Sommerfeld enhancement will saturate at velocity dispersions of the order of the velocity dispersion of the dwarf Spheroidals. This means that the specific form that we adopt below for the velocity dispersion-mass scaling relation does not matter greatly.

On the scale of clusters there is a well-defined relationship between the mass of a DM halo and its onedimensional velocity dispersion which one can derive from adiabatic scaling arguments [118] given by

$$
\sigma \propto m^{1 / 3} .
$$

This scaling relation can be easily derived in the case of an isothermal distribution function [137], since then

$$
\rho(r)=\frac{\sigma^{2}}{2 \pi G r^{2}},
$$

from which equation (34) immediately follows. This scaling relation has been found to describe numerical simulations of cluster-sized halos extremely well $[77,82,118$, 138, 139, 140]. From recent numerical simulations it seems that we can use this relation even for subhalos of subhalos in a Galaxy sized dark matter halo. In Ref. [61] it is shown that the relation between the maximum circular velocity of a subhalo and its mass is well fit by a power-law of the type

$$
m \propto v_{\max }^{3.5} .
$$

Using the circular velocity at $r_{-2}$ as a measure of the halo's velocity dispersion gives $\sigma_{v} \propto m^{1 / 3.5}$. Alternatively, we could use the circular velocity at the virial radius as a measure of the velocity dispersion. In the case of an NFW profile we have that

$$
\frac{v_{\max }^{2}}{v_{\mathrm{c}}\left(r_{\mathrm{vir}}\right)^{2}} \propto \frac{c}{f(c)}
$$

in which $f(x)=\ln (1+c)-c /(1+c) \approx 2.6(c / 33)^{0.4}$ in the relevant concentration range. Using equation (33), this gives

$$
\sigma_{v} \propto m^{0.32} .
$$

Therefore, we will use (as a rough approximation) the scaling relation given in equation (34). We calibrate this relationship at the mass scale of the dwarf spheroidals by using the velocity and mass of Canes Venatici I from Ref. [70].

\section{The total and the "reduced" boost}

We define the total boost for a given subhalo mass as (see equation [29])

$$
\mathcal{B}(M)=S(M)+B(M),
$$

in which $S(M)$ is the Sommerfeld enhancement for a halo of mass $M$ and $B(M)$ is the substructure boost for a halo of that mass, as defined in Eq. (30). This total boost for subhalos of mass $M=10^{8} M_{\odot}$ and $M=10^{7}$ $M_{\odot}$ calculated by following the procedure outlined in the previous subsection is shown in Figure 5 (top panels). It is immediately obvious that the total boost factors can get very large in certain regions of the parameter space spanned by $m_{\phi} / m_{\chi}$ and $\alpha$ : boosts vary between $10^{2}$ to $10^{9}$. These masses correspond to the typical masses of the classical and newly-discovered dwarf Spheroidals (dSphs) and as such their velocity dispersions are of the order $10^{-5}$. The pattern of resonance regions and nonresonance regions that appears for the pure Sommerfeld enhancement (as shown in Figure 2) is also apparent in these plots of the total boosts, however, it is noteworthy that the resonances seems to be more highly peaked than they were in the pure Sommerfeld enhancement case, and that the boost the lower half of the parameter space, i.e., for smaller values of $m_{\phi} / m_{\chi}$, are higher than one would expect from the pure Sommerfeld enhancement combined with a boost factor from pure density enhancements.

This is confirmed by looking at the "reduced boost" defined as

$$
\mathcal{B}_{\text {red }}(M) \equiv \frac{\mathcal{B}(M)}{S(M)\left(1+B_{0}(M)\right)},
$$

in which $B_{0}(M)$ is the boost we would find if we turned off the Sommerfeld enhancement in the calculation of the total boost, i.e., the boost we would get if we set $S(m)$ equal to one in equation (30). This $B_{0}$ corresponds to the boost factor that has been used before to estimate the total boost from density enhancements in dSphs and it is generally $\mathcal{O}(10)$. This reduced boost therefore divides out the contribution of the Sommerfeld enhancement of the top-level subhalo and the boost from the overdensities in lower-level halos, and it would be equal to one if there were no extra boost coming from the fact that the substructures are kinematically colder than the main subhalo. As we can see in Figure 5 (bottom panels), in various parts of the Yukawa coupling parameter space this reduced boost is equal to one such that there is no extra boosts caused by the lower velocity dispersion in the substructures. This is mainly the case for large values of the parameter $m_{\phi} / m_{\chi}$, since the Sommerfeld enhancement, in the $1 / v$ regime, levels off around $S \sim m_{\phi} / m_{\chi}$, and since the top-level subhalo has a velocity dispersion of $\sim 10^{-5}$ this means that the Sommerfeld enhancement has mostly saturated at these large values of $m_{\phi} / m_{\chi}$, even in regions of parameter space in which $\alpha$ is small. Indeed, in the $1 / v$ regime the Sommerfeld enhancement is $\sim \alpha / \beta \approx 10^{2}$ for $\alpha=10^{-3}$, such that the Sommerfeld enhancement has fully saturated over the whole $\alpha$ range for large values of $m_{\phi} / m_{\chi}\left(m_{\phi} / m_{\chi} \sim 10^{-2}\right)$.

However, the Sommerfeld enhancement has not saturated in all regions of parameter space at this mass scale, and for smaller values of $m_{\phi} / m_{\chi}$ and especially in resonance regions, the reduced boost can reach high values, 

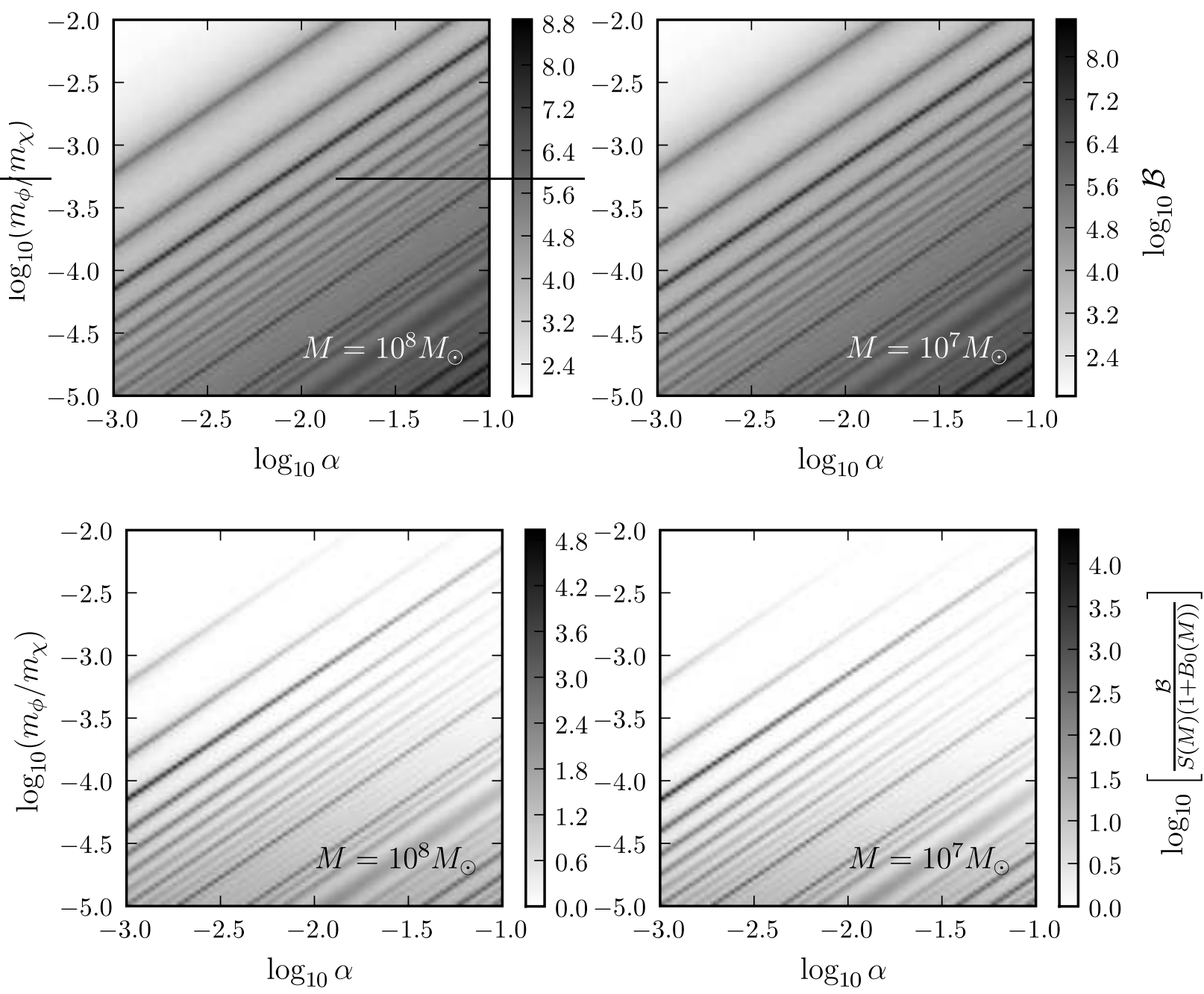

FIG. 5: The total (defined in Eq. [39]) and reduced boost (defined in Eq. [40]) for subhalos of two different mass scales. The dark matter profile of all the subhalos is assumed to be well-described by an NFW profile.

signaling a large extra boost caused by the kinematically coldness of the substructure. For small values of $m_{\phi} / m_{\chi}$ we see that we can get boosts that are an order of magnitude to two orders of magnitude larger than what we would expect from naively combining the Sommerfeld enhancement from the velocity dispersion of the subhalo with the boost from density enhancements alone. This can be easily understood as being a consequence of the fact that the Sommerfeld enhancement has not saturated yet at the mass scale of the top-level subhalo, such that it can be boosted up to it saturation point by the contribution from the kinematically colder substructure.

The largest values of the reduced boost are found in the resonance regions. In these regions the Sommerfeld enhancement both grows faster with decreasing relative velocity as well as saturates at a much larger value of the enhancement. Both of these effects conspire to give very large extra boosts. The resonance regions are clearly distinguishable in this plot of the reduced boost as $45^{\circ}$ lines.
Because the resonance regions are so narrow the apparent extra boosts in different resonances seems to vary greatly, but this is mostly caused by the finite resolution of this figure. Extra boosts up to $\sim 10^{5}$ are obtained in these resonances, and these extra boosts increase along the resonances when decreasing $\alpha$, again caused by the higher saturation level for smaller $m_{\phi} / m_{\chi}$ values.

The total boost factors obtained by assuming an NFW dark matter density profile do not depend much on the inner slope of the density profile. This is mainly because the calculation of the boost only considers relative luminosities, for which the difference in normalization of the $\mathcal{L} \propto \rho_{s}^{2} r_{s}^{3} / D^{2}$ relation for different inner slopes is irrelevant, and because the mass of the subhalo is only weakly affected by the value of the inner slope - the function $f(c)$ is only a weak function of the inner slope. This is confirmed by calculating the total boost for a $M=10^{8} M_{\odot}$ subhalo for a subhalo with a very shallow inner cusp as well as for a subhalo with a very steep inner slope. These 

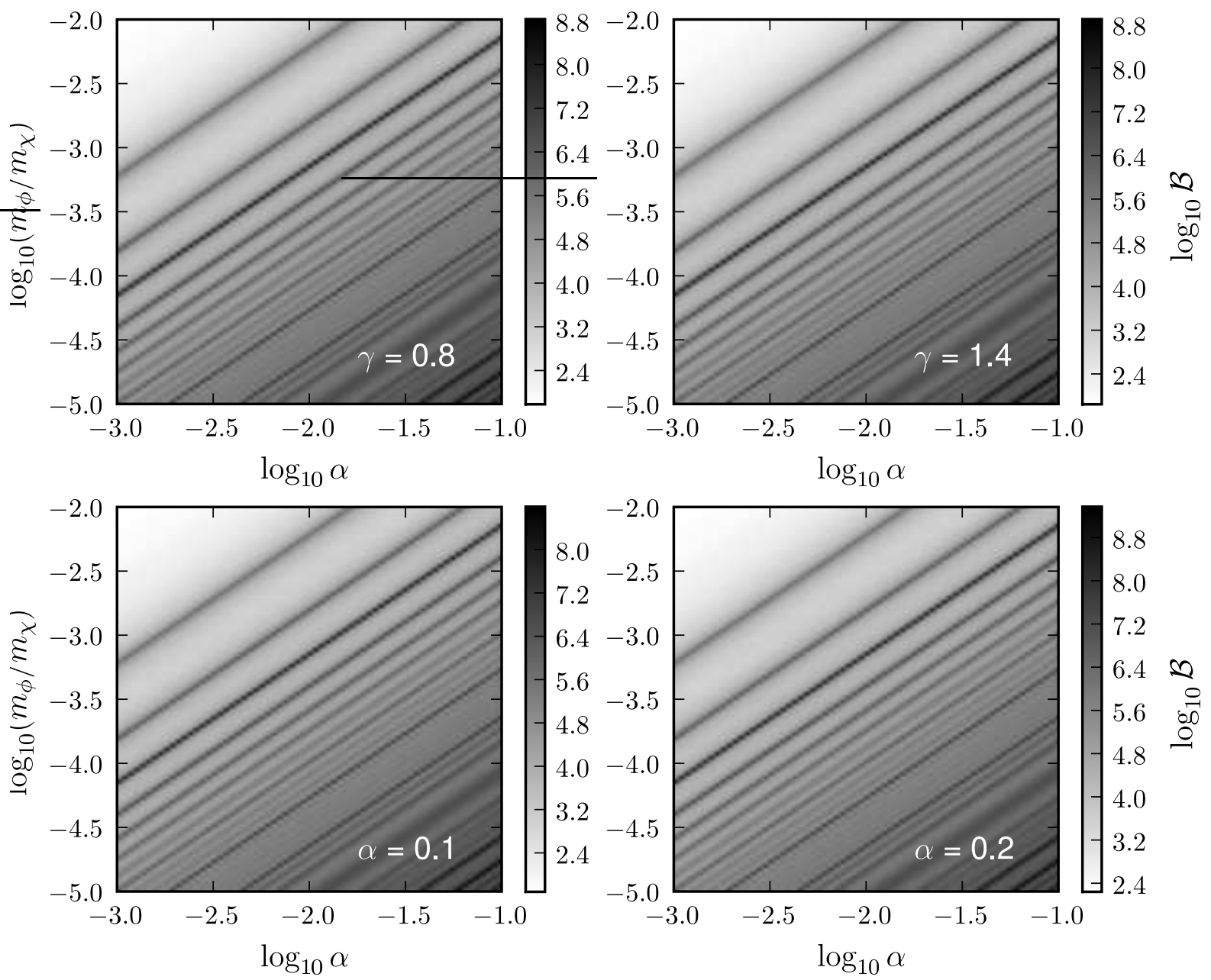

FIG. 6: The total boost for a $M=10^{8} M_{\odot}$ subhalo for different shapes of the DM density profile: a generalized NFW profile described by an inner slope $\gamma$ (top panels); an Einasto profile described by a shape parameter $\alpha$ (bottom panels).

are shown in Figure 6 (top panels), and, from comparing this with the total boost shown in Figure 5, it is clear that the inner slope does not affect the total boost caused by substructure, neither the overall magnitude of the effect nor the structure in the $\left(m_{\phi} / m_{\chi}, \alpha\right)$ plane. The main difference in DM annihilation flux between the different dark matter density profiles is therefore given by the normalization of the $\mathcal{L} \propto \rho_{s}^{2} r_{s}^{3} / D^{2}$ relation, shown in Figure 3.

If the DM density profiles of halos is better described by an Einasto profile then the substructure boost is affected more strongly by the shape parameter $\alpha$ which basically describes how cored the DM density profile is. Changing $\alpha$ has a greater impact on changing the overall DM density profile and therefore also plays a larger role in the relation between the characteristic density, the concentration, and the mass of the subhalo. The total boosts for a $M=10^{8} M_{\odot}$ subhalo is shown in Figure 6 (bottom panels) for two different values of the shape parameter $\alpha$ which span the range found in numerical simulations (for values of $\alpha$ greater than 0.2 the behavior basically saturates at that of $\alpha=0.2$ ). For $\alpha=0.1$ the total boost is about the same as for the GNFW profile shape, which is understandable given that the Einasto profile is more cuspy for smaller values of $\alpha$. The same dependence on the parameter $m_{\phi} / m_{\chi}$ as is seen for the GNFW profile is displayed for the Einasto profile. In the case that $\alpha$ is larger the substructure boost reaches slightly larger values than for the GNFW density profile. Thus, as was the case for the GNFW profile, the impact of the different normalization of the $\rho_{-2}^{2} r_{-2}^{3} / D^{2}$ for different values of $\alpha$ will play a leading role in the difference between the different profile shapes.

In Figure 7 the effect of varying two more of the many parameters introduced in the previous section is shown. The slope of the subhalo mass function $n$ was set to 0.9 before, corresponding to the results from the latest high-resolution numerical simulations. In Figure 7 (top panels) we show the effect of both a shallower as well as a steeper subhalo mass function. The value of $n=-0.7$ corresponds to the shallow slope found in Ref. [125]. We see that the effect of such a shallow slope is to seriously 

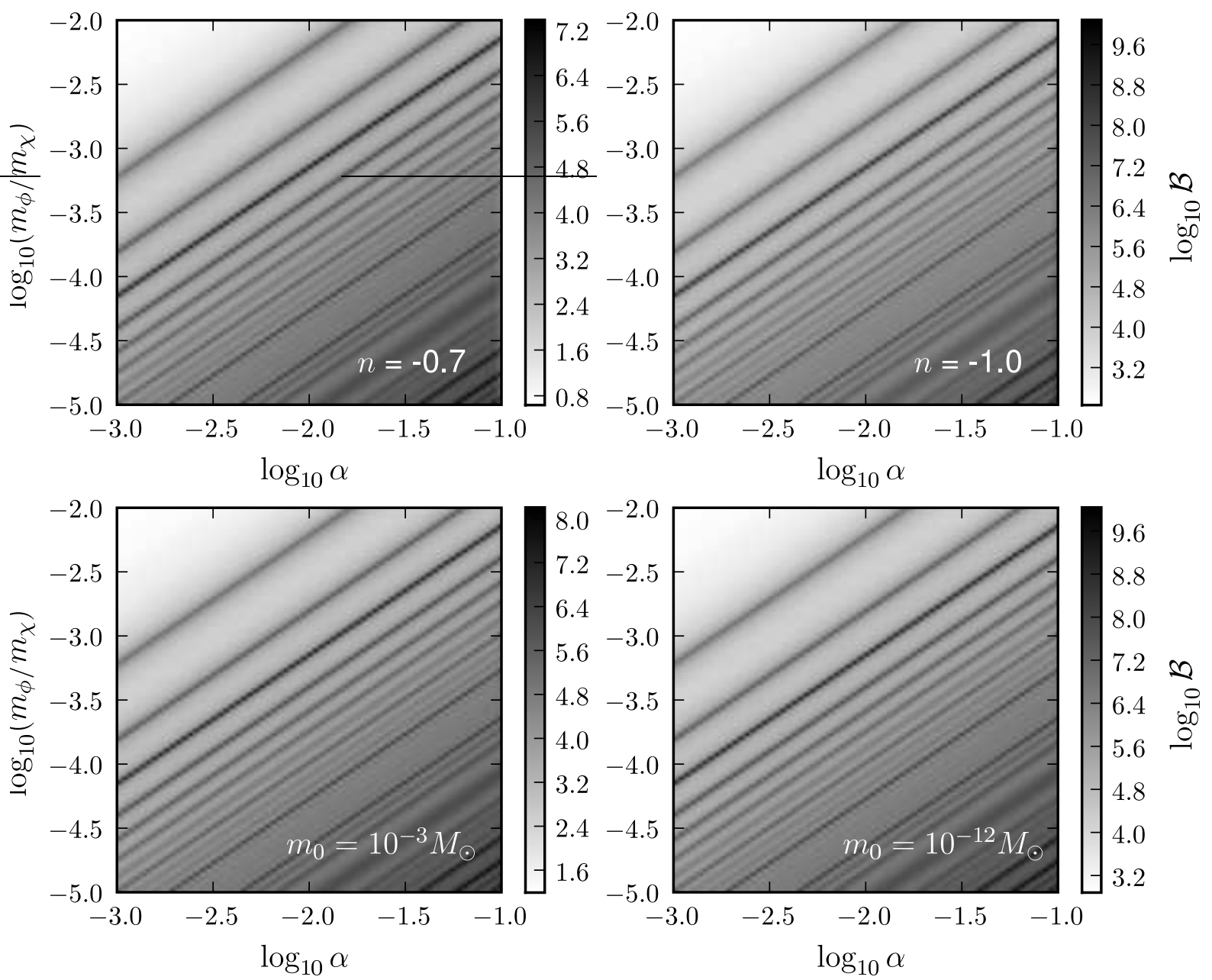

FIG. 7: The total boost for a $M=10^{8} M_{\odot}$ for different slopes $n$ for the subhalo mass function (top panels) and for different values of the dark matter thermal free streaming limit $m_{0}$ (bottom panels).

\begin{tabular}{|c|c|c|c|c|c|c|c|c|}
\hline dSph & $\begin{array}{r}D \\
{[\mathrm{kpc}]}\end{array}$ & {$\left[\begin{array}{c}\sigma_{0} \\
{\left[\mathrm{~km} \mathrm{~s}^{-1}\right]}\end{array}\right.$} & $\begin{array}{l}r_{\max } \\
{[\mathrm{kpc}]}\end{array}$ & $\begin{array}{c}v_{\max } \\
{\left[\mathrm{km} \mathrm{s}^{-1}\right]}\end{array}$ & $\begin{array}{c}\rho_{s} \\
{\left[M_{\odot} \mathrm{kpc}^{-3}\right]}\end{array}$ & $\begin{array}{c}r_{s} \\
{[\mathrm{kpc}]}\end{array}$ & $\begin{array}{c}M\left(<r_{s}\right) \\
{\left[M_{\odot}\right]}\end{array}$ & References \\
\hline Carina................. & 101 & 6.8 & 3 & 16 & $1.1 \times 10^{7}$ & 1.4 & $7.4 \times 10^{8}$ & 1,3 \\
\hline Coma Berenices.... & 44 & 4.6 & . & . & $3.0 \times 10^{8}$ & 0.3 & $2.0 \times 10^{7}$ & 2,4 \\
\hline Draco.......................... & 80 & 5.5 & 8 & 40 & $1.0 \times 10^{7}$ & 3.7 & $1.2 \times 10^{9}$ & 1,3 \\
\hline Fornax............ & 138 & 11.1 & 4 & 20 & $1.0 \times 10^{7}$ & 1.9 & $1.5 \times 10^{8}$ & 1,3 \\
\hline Leo I...... & 250 & 8.8 & 6 & 30 & $1.0 \times 10^{7}$ & 2.8 & $5.2 \times 10^{8}$ & 1,3 \\
\hline 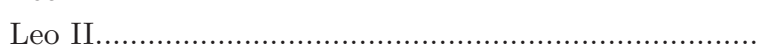 & 205 & 6.7 & 4 & 20 & $1.0 \times 10^{7}$ & 1.9 & $1.5 \times 10^{8}$ & 1,3 \\
\hline 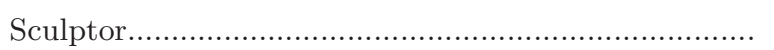 & 79 & 8.5 & 6 & 30 & $1.0 \times 10^{7}$ & 2.8 & $5.2 \times 10^{8}$ & 1,3 \\
\hline Sextans................ & 86 & 5.8 & 2 & 10 & $1.0 \times 10^{7}$ & 0.9 & $1.9 \times 10^{7}$ & 1,3 \\
\hline 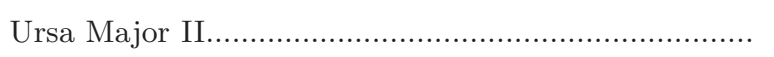 & 32 & 6.7 & & & $3.0 \times 10^{8}$ & 0.3 & $2.0 \times 10^{7}$ & 2,4 \\
\hline 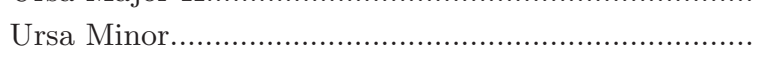 & 66 & 15.0 & 6 & 30 & $1.0 \times 10^{8}$ & 2.8 & $5.2 \times 10^{8}$ & 1,3 \\
\hline
\end{tabular}

TABLE I: Properties of the dwarf Spheroidals used in this study.

References: (1) Peñarrubia et al. [141], (2) Strigari et al. [67], (3) Mateo [69], (4) Simon and Geha [70].

reduce the substructure boost, which is simply a consequence of the fact that for a shallower slope less substruc- ture is found in smaller and more concentrated clumps. A steeper slope such as $n=-1$, as was found in the 
Carina

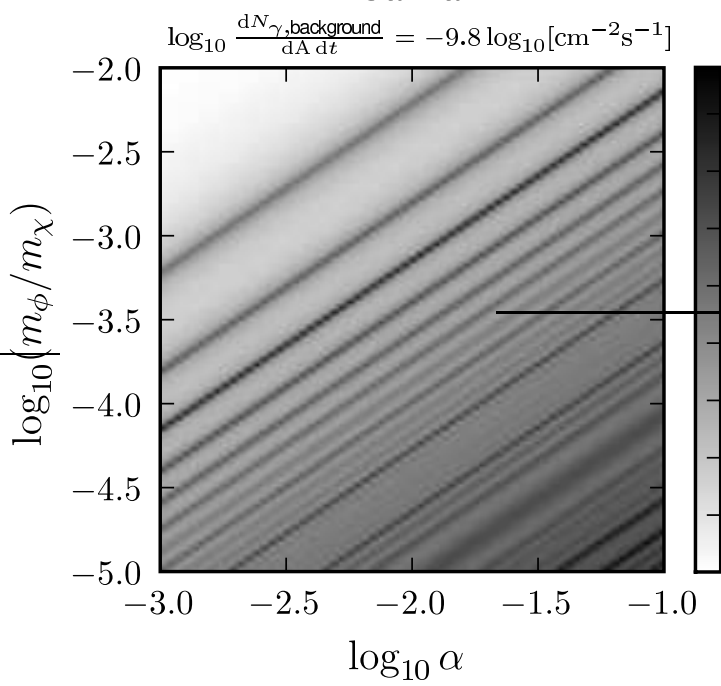

Fornax

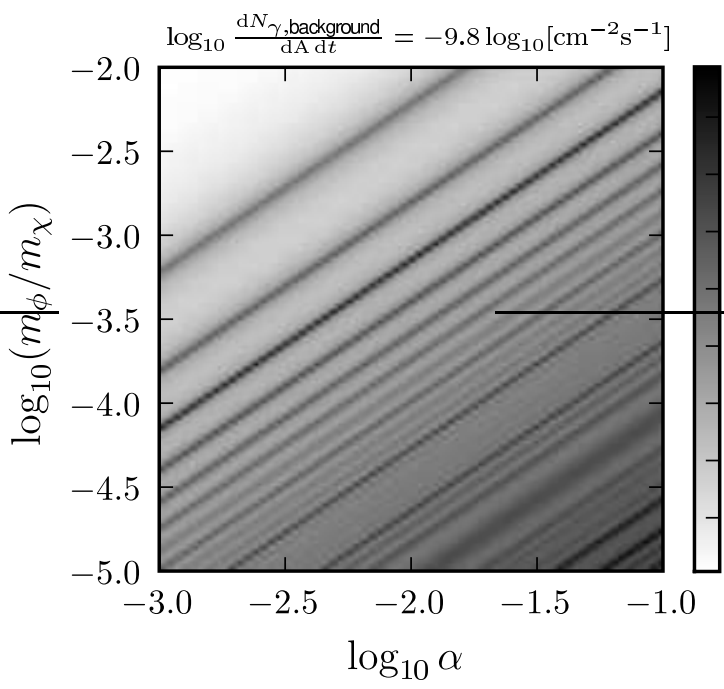

Ursa Major II

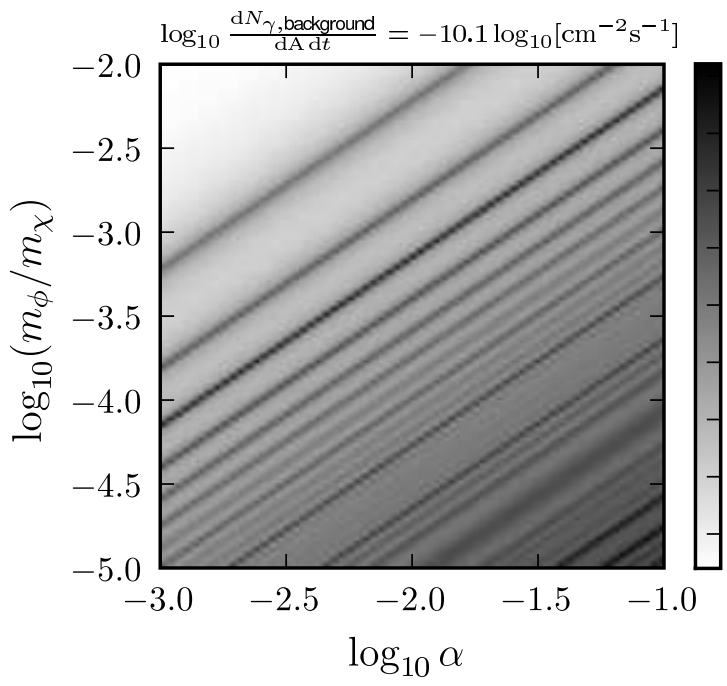

Draco

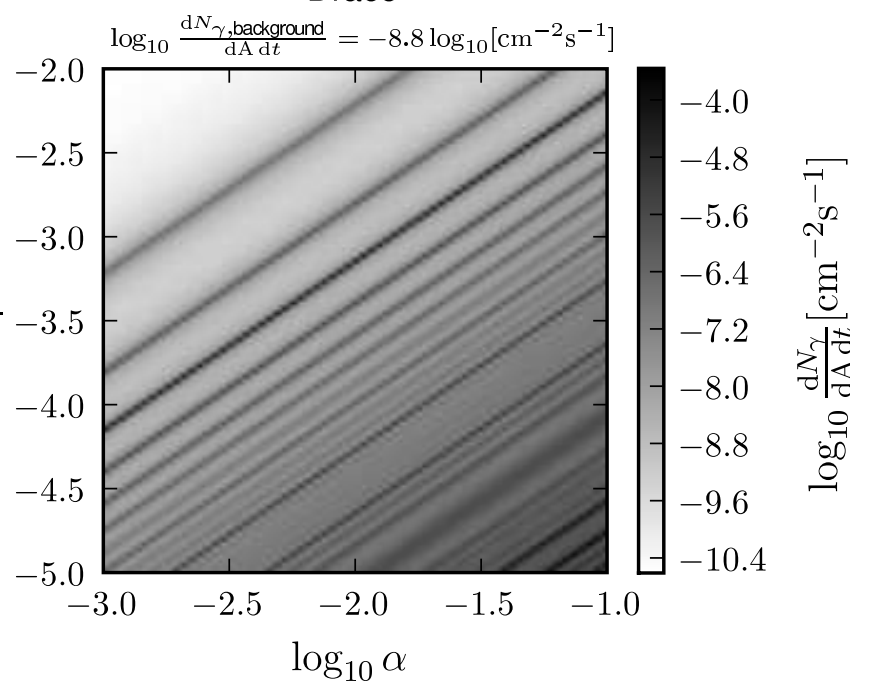

Sextans

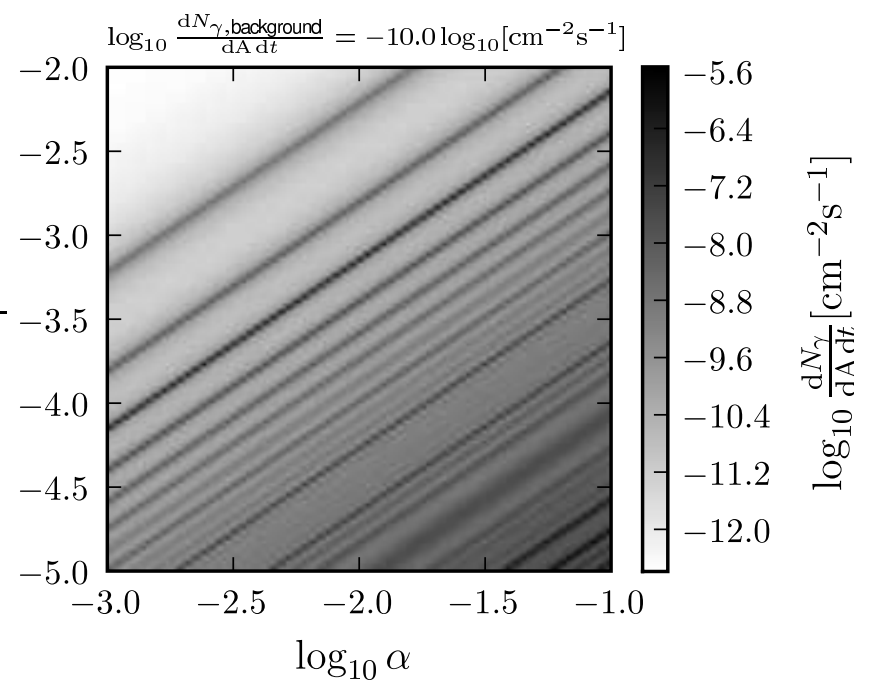

Ursa Minor

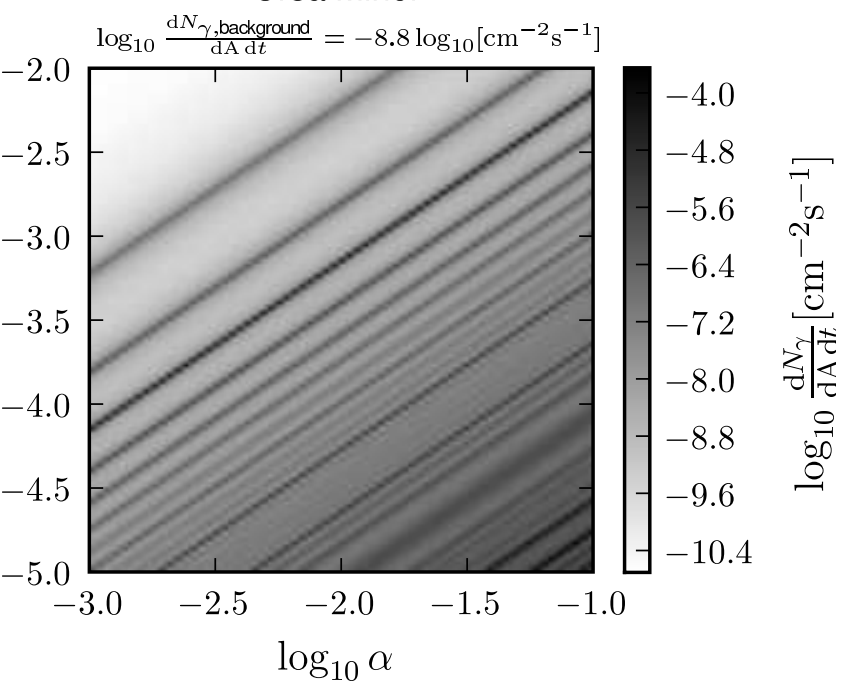

FIG. 8: Predicted DM annihilation fluxes for various dwarf Spheroidal satellite galaxies. The background flux of extragalactic photons in the relevant energy range is given under the name of the dwarf Spheroidal. The properties of the dSphs used are given in Table I. 
recent Via Lactea simulation [6] leads to a substructure boost which is about an order of magnitude larger than for the fiducial $n=-0.9$ value.

The effect of the thermal free-streaming scale of DM on the substructure boost is shown in Figure 7 (bottom panels). This effect is relatively large since the freestreaming scale sets the scale of the smallest and most concentrated DM halos and raising or lowering this scale therefore changes an important part of the contribution to the substructure boost. For very large values of the free-streaming scale such as $m_{0}=10^{-3} M_{\odot}$, the substructure boost becomes about an order of magnitude smaller. When the free-streaming scale becomes very small, much of the substructure is in very small, cold clumps, such that the total boost becomes about an order of magnitude larger.

\section{PREDICTED ANNIHILATION SIGNALS FOR DWARF SPHEROIDALS}

Besides detection of DM annihilation from the Galactic Center or the diffuse component from the Galactic halo, there idea exists that the best candidates for DM annihilation detection might be the dwarf Spheroidal satellite galaxies (dSphs) of our Galaxy, since the dwarf galaxies are believed to be the most dark matter dominated structures in the Universe, and the DM annihilation signal coming from them would be much easier to distinguish from background sources than, e.g., would be the case for DM annihilation photons coming from the Galactic Center. That dSphs make good candidates for DM annihilation detection has been much discussed before, especially for the "classical", or pre-SDSS, dSphs $[62,110,142,143,144,145]$. The annihilation signal coming from Draco has been the subject of much research in recent years because of its relative proximity to the Earth $[65,110,144,146,147,148,149,150]$. The prospects for DM annihilation detection from dSphs has in general been found to be rather poor, with detection with Fermi being unlikely $[150,151]$. Some of the dSphs have already been the subject of observational searches for DM annihilation and some limits on the DM annihilation signal have already been set $[152,153,154,155,156,157]$.

The dSphs discovered by SDSS have also been suggested to make good candidates for DM annihilation detection, because of their very large mass-to-light ratios $\left(\sim 10^{3}\right)[67,68]$. In the context of the Sommerfeld enhancement, these recently discovered dSphs are especially interesting, since they also have the smallest velocity dispersion of all of the known satellites of our Galaxy. Predicting the DM annihilation signal from these lowsurface brightness galaxies is complicated by the dearth of kinematical tracers of the DM distribution and their contamination with background stars [158]. Nevertheless, detailed studies have constrained the DM density and velocity dispersion profiles of some of these dSphs $[65,73,141]$. We will use some of the results on the
DM density and velocity profiles found in these studies to show the extra effect of the Sommerfeld enhancement on the predicted DM annihilation signal from some of these dSphs.

We consider the dSphs given in Table I here. Eight of the dSphs are classical dwarf galaxies and we use the DM density profiles fitted to them in Ref. [141]. In this the DM density profiles of the dSphs were not completely determined by the available data - a degeneracy between the parameters of the NFW profile used to describe the DM density and the King profile used to fit the luminous component of the galaxies was found - and the concentration-mass relation from CDM [71, 72] was used to break the degeneracy. Since these authors expressed their results in the form of the $r_{\max }$ and $v_{\max }$ parameters of the DM density profiles, we use the procedure outlined in Section III B to convert these to $\rho_{s}$ and $r_{s}$ values. As these $v_{\max }$ and $r_{\max }$ parameters were fit to an NFW DM profile, we will only consider an NFW profile for the dSphs here, but the general conclusions about the importance of the exact profile shape also hold in these particular cases. The velocity dispersions of these dSphs are taken from Ref. [69] and we again model these subhalos as having a constant velocity dispersion.

We also consider some of the newly discovered dSphs. In particular we discuss the prospects for DM annihilation detection from Coma Berenices and Ursa Major II, and we use the best fit values to the available data from Ref. [67]. Since these authors directly quote $\rho_{s}$ and $r_{s}$ values we do not include $r_{\max }$ and $v_{\max }$ values for these dSphs. We use the velocity dispersions determined for these galaxies in Ref. [70].

In order to calculate the substructure boost to the DM annihilation cross section we need the mass of the dSphs. Many definitions of the mass of the galaxy could be used here, among the more appropriate ones is the mass contained inside the tidal radius, i.e., the radius inside of which material in the $\mathrm{dSph}$ is safe from being tidally stripped by the Galaxy. However, since we are considering the DM annihilation flux coming from within the radius $r_{-2}$ we will use the mass contained within this radius to calculate the substructure boost. This is more appropriate since (1) it can be calculated straightforwardly from the DM density profile of the dSph and (2) the mass contained within $r_{-2}$ is really the more relevant mass to calculate the substructure boost, since we are only interested in contributions to the DM annihilation flux coming from within the radius $r_{-2}$.

Because of the similarities between some of the DM density profiles of the dSphs in Table I we do not show the predicted annihilation fluxes for all of the dSphs in Figure 8, instead focusing on a representative sample of them. Inspection of Table I shows that annihilation flux of the dSphs not shown here will be similar to that of the ones shown in Figure 8.

At this point we can also make a connection to another quantity that is often used to describe the astrophysical contribution to the DM annihilation signal, J. This 
quantity is generally defined as

$$
J \equiv \frac{1}{\Delta \Omega} \int_{\Delta \Omega} \mathrm{d} \Omega \int_{\operatorname{los}} \mathrm{d} l \rho^{2},
$$

and is therefore equal to the line-of-sight integral over the density squared averaged over solid angle. We can define a slight variant of this which takes into account the Sommerfeld enhancement

$$
\tilde{J} \equiv \frac{1}{\Delta \Omega} \int_{\Delta \Omega} \mathrm{d} \Omega \int_{\operatorname{los}} \mathrm{d} l \rho^{2} S,
$$

in which $S$ is the Sommerfeld enhancement averaged over the distribution of relative velocities. If we work this out for the dwarf Spheroidals, using the expression from equation (14) for the line-of-sight integral averaged over the solid angle spanned by the scale radius $r_{s}$ we find that this $\tilde{J}$ is approximately equal to the total boost $\mathcal{B}$, with a pre-factor $\mathcal{O}(1)$. Therefore in the case of the dSphs studied here the $\tilde{J}$ can be approximately read off from the top panels of Figure 5 .

It is clear from Figure 8 that the DM annihilation flux predicted to be coming from the dSphs is very large in a large class of models. We have to stress here that our predicted fluxes are all for the particle physics specific parameters given in Section III A, i.e., $\langle\sigma v\rangle=3 \times 10^{-26} \mathrm{~cm}^{3}$ $\mathrm{s}^{-1}, m_{\chi}=700 \mathrm{GeV}$ and a particular form for the DM annihilation spectrum. To predict the gamma-ray flux for a particular model with different values of these particle physics parameters one is required to calculate the particle physics parameter specific factor to the DM annihilation flux and appropriately rescale the values given here. In order to compare these signals to the expected background flux we will only consider contributions to the background flux of extragalactic gamma rays. Although there are also contributions to the background flux from pion-decay, inverse Compton scattering, and Bremsstrahlung, at the high Galactic latitudes at which the dwarf Spheroidals are found these are all of the same order or smaller than the extragalactic background [159]. We have integrated the extragalactic gamma ray flux as measured by EGRET over the energy range between 4 $\mathrm{GeV}$ to $250 \mathrm{GeV}$ and multiplied it with the solid angle that the dSphs span on the sky. The extragalactic gamma ray flux is given by a power law [160]

$$
\frac{\mathrm{d} N_{\gamma}}{\mathrm{d} E \mathrm{~d} A \mathrm{~d} t \mathrm{~d} \Omega}=k\left(\frac{E}{E_{0}}\right)^{-\alpha},
$$

where $k=7.3 \times 10^{-6} \mathrm{~cm}^{-2} \mathrm{~s}^{-1} \mathrm{sr}^{-1} \mathrm{GeV}^{-1}, \alpha=$ 2.10 , and $E_{0}=0.45 \mathrm{GeV}$. We have included the background flux of extragalactic photons in Figure 8. This background flux is in all cases of the order of $10^{-9}$ to $10^{-10}$ photons $\mathrm{cm}^{-2} \mathrm{~s}^{-1}$, such that the DM annihilation boosted by substructure and Sommerfeld enhancement outshine this background in most of the parameter space, and by a few orders of magnitude in most models. Given that the flux above $100 \mathrm{MeV}$ over a 55 days time-span
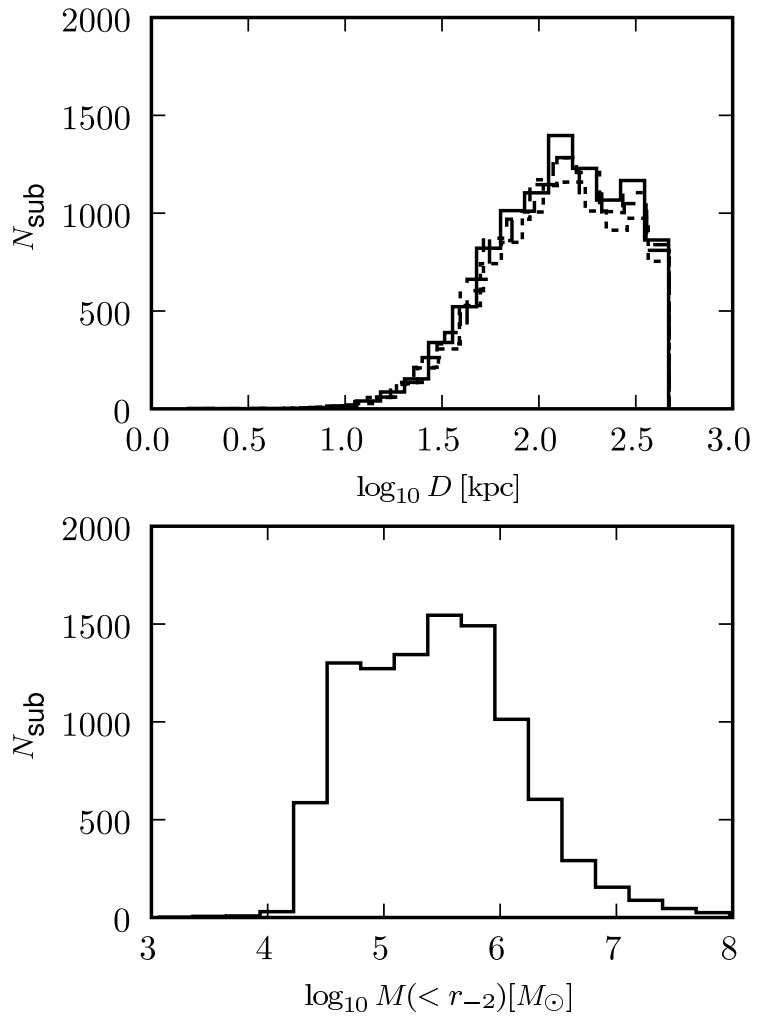

FIG. 9: The distances to and masses of subhalos within the tidal radius of the main halo in Via Lactea II. The distances are for three random observers at a distance of $8.5 \mathrm{kpc}$ from the galactic center of the main halo.

needed to separate a point source of DM annihilation from the background by Fermi to give a $5 \sigma$ detection is about $10^{-8}$ photons $\mathrm{cm}^{-1} \mathrm{~s}^{-1}$ and that the necessary fluxes scale roughly as $t_{\exp }^{-1 / 2}$ [114], the DM annihilation of these dSphs should be detectable by Fermi in many of the models parametrized by $\left(m_{\phi} / m_{\chi}, \alpha\right)$. The Ursa Major II dSph is the only dSph for which the DM annihilation dominates the background in all the regions of parameter space. This shows that the newly discovered, most-dark-matter-dominated satellite galaxies are possibly the best targets for the indirect detection of DM. We conclude that the prospects for detecting DM annihilation from the dSphs with Fermi are very good in models that include a significant Sommerfeld enhancement.

\section{THE ANNIHILATION SIGNAL FROM SUBHALOS IN VIA LACTEA II}

Besides the satellite galaxies that have been observed in the Galactic halo CDM predicts that the halo is filled with hundreds of individual subhalos - a fact which gives rise to the so-called "missing satellites problem" $[57,58]$. 

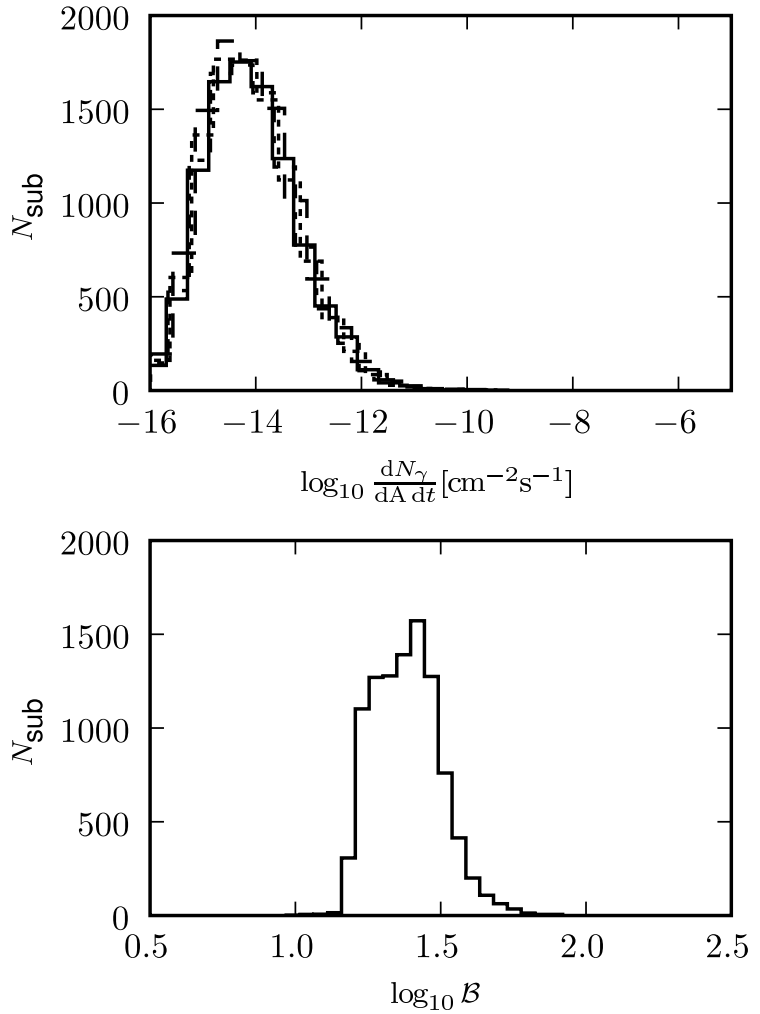

FIG. 10: Top: Histogram of the predicted gamma-ray flux coming from individual subhalos contained within the tidal radius of the main halo in Via Lactea II for three random observer placed at a distance of $8.5 \mathrm{kpc}$ from the center of the main Via Lactea halo for $m_{\phi} / m_{\chi}=10^{-2}$ and $\alpha=10^{-3}$. Bottom: Histogram of the total boosts of the subhalos for the same values of $m_{\phi} / m_{\chi}$ and $\alpha$.

Each of these subhalos has a mass of the order of the masses of the observed dSphs or smaller, and thus will be kinematically cold with respect to the main halo. Thus, the total DM annihilation signal coming from all of the substructure will be boosted by the Sommerfeld enhancement described above, such that an appreciable DM annihilation signal could be expected from all of the substructure. In this section we get a feeling for the expected signal from DM substructures in a typical Galaxy-sized halo. If the DM annihilation signal from subhalos without star formation could be detected this could give a clear signal that the DM exists and would open up a whole new arena for testing CDM.

We use the distribution of DM subhalos from the Via Lactea II numerical $N$-body simulation ${ }^{1}[38]$. Via Lactea II is a state-of-the-art $N$-body simulation of a Galaxy-

\footnotetext{
${ }^{1}$ Available at http://www.ucolick.org/ diemand/vl/data.html
}

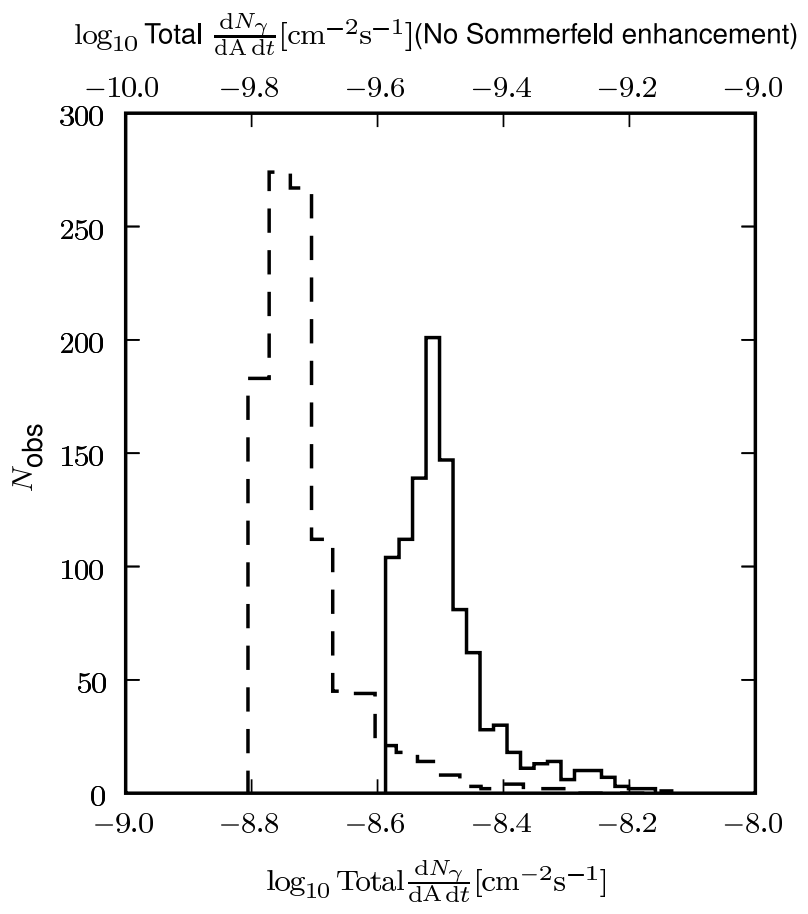

FIG. 11: Total gamma-ray flux from subhalos in Via Lactea II for 1,000 random observers at $8.5 \mathrm{kpc}$ from the center of the main Via Lactea halo for $m_{\phi} / m_{\chi}=10^{-2}$ and $\alpha=10^{-3}$. For comparison we show the total gamma-ray flux from subhalos in the absence of Sommerfeld enhancement as the dashed histogram and the top horizontal axis.

sized halo since redshift 104.3. It sampled the region of the main halo with $1.1 \times 10^{9}$ particles, each with a mass of 4,100 $M_{\odot}$. Over 40,000 subhalos can be resolved within $402 \mathrm{kpc}$ of the center of the main halo and 20,047 subhalos are detected which had a peak circular velocity larger than $4 \mathrm{~km} \mathrm{~s}^{-1}$ at some time. The properties of the subhalos in this latter sample can be accurately established, i.e., they are unaffected by resolution effects. We select from this sample the 9,830 subhalos which lie within the tidal radius of the main halo, which is approximately $462 \mathrm{kpc}$. We show the distribution of these subhalos in mass and distance from the Sun in Figure 9. We have placed the Sun at random locations at a distance of $8.5 \mathrm{kpc}$ of the center of the main halo, corresponding to an earth-based-observer-like vantage point. The distribution of the distances in Figure 9 is given for three of these random observers and the distribution is very similar for all three observers. We see that most of the subhalos have masses in the range $10^{4}$ to $10^{6} M_{\odot}$, with subhalos with masses of the order of the dSphs being relatively rare. The lower limit is of course set by the finite numerical resolution of the simulation and doesn't correspond to a physical cut-off-indeed, the physical cut-off lies at the thermal free-streaming scale. The distribution of the distance to the subhalos shows that the distribution of the subhalos is strongly radially anti-biased, with a sharp cut-off caused by our cut at the tidal radius of the main halo. This radial anti-bias is a consequence of 

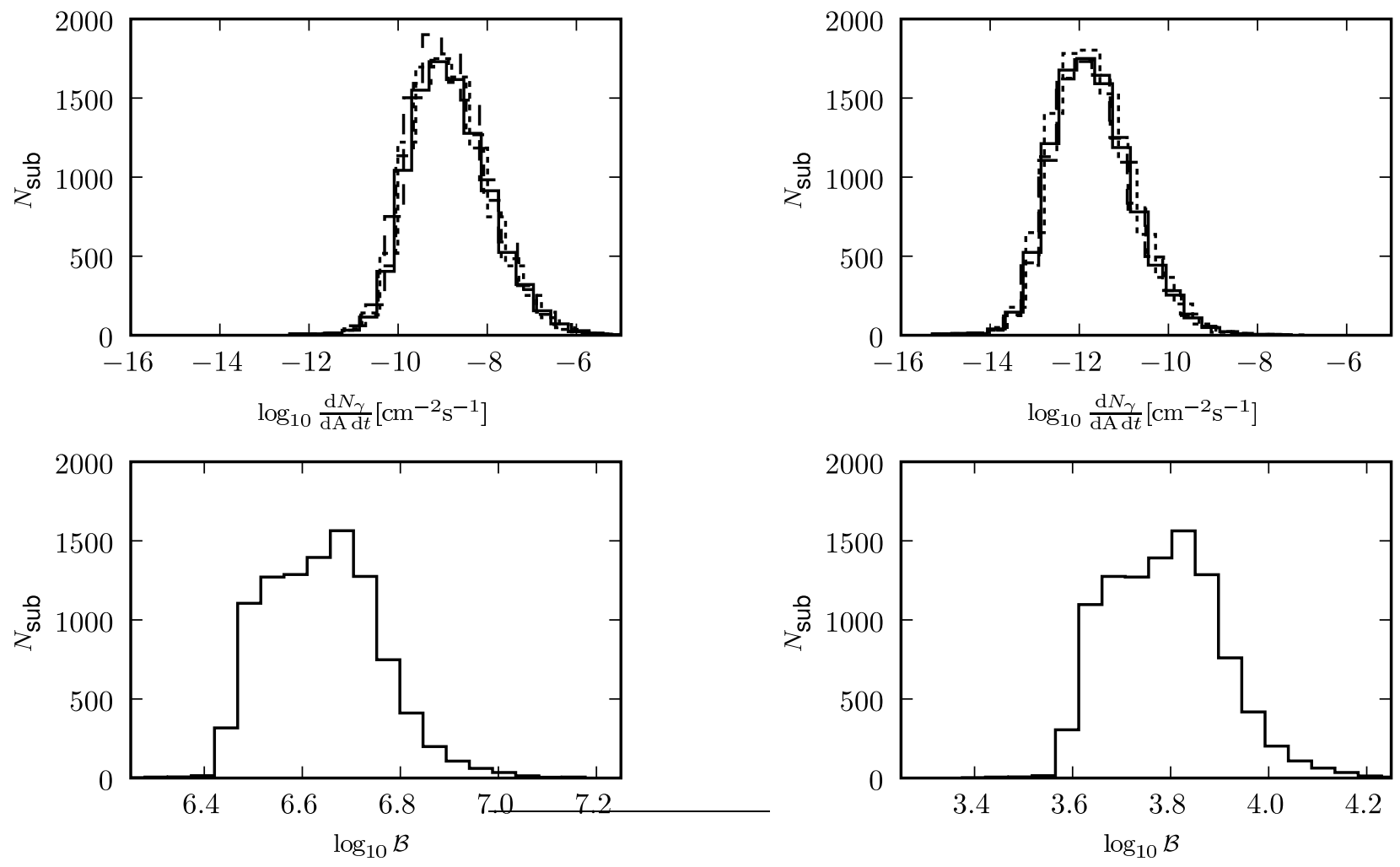

FIG. 12: Same as Figure 10 but for $m_{\phi} / m_{\chi}=10^{-5}$ and $\alpha=10^{-1}$.

FIG. 14: Same as Figure 10 but for a resonance: $m_{\phi} / m_{\chi}=$ $10^{-3.84375}$ and $\alpha=10^{-2.625}$.
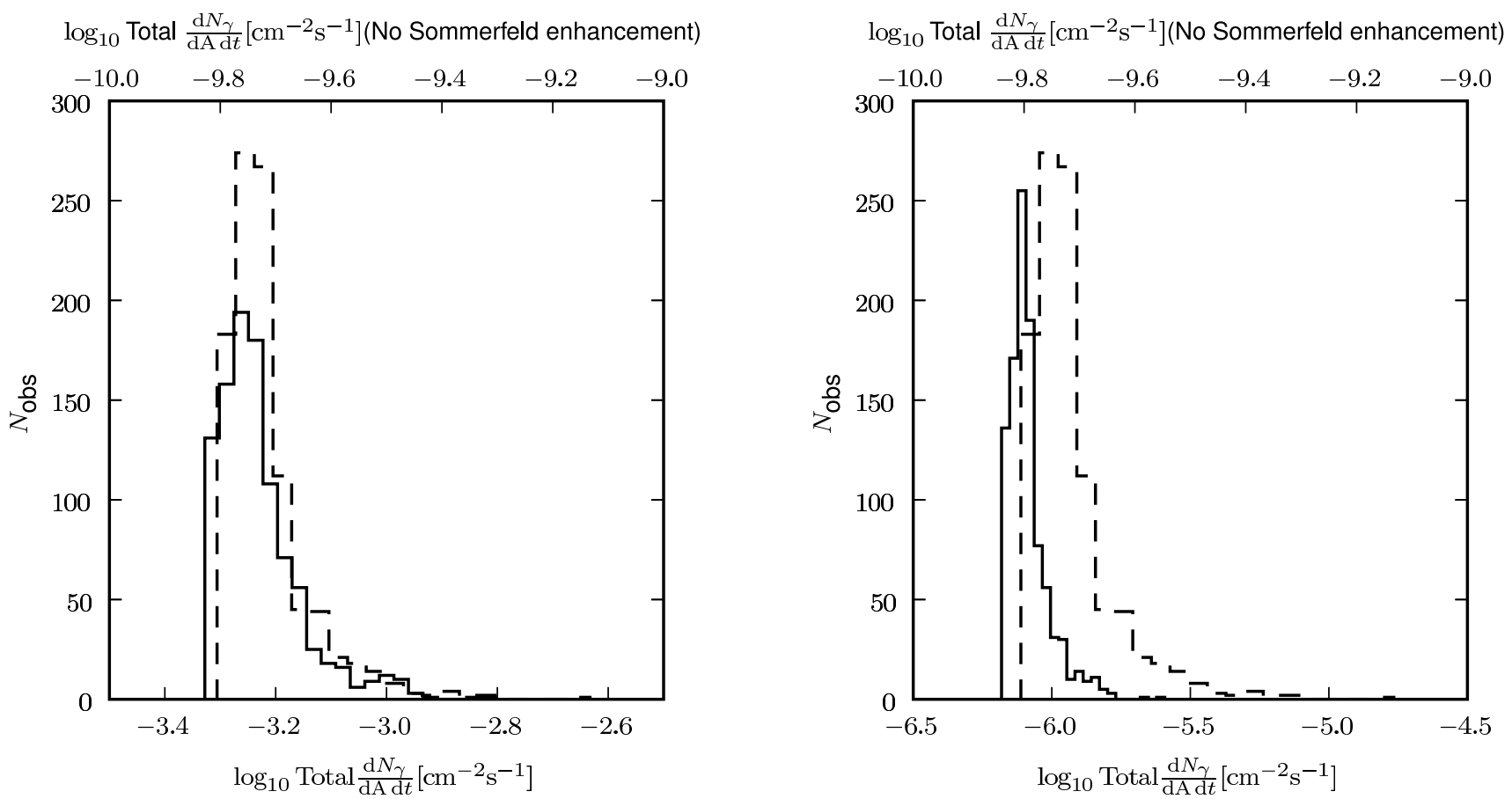

FIG. 13: Same as Figure 11 but for $m_{\phi} / m_{\chi}=10^{-5}$ and $\alpha=10^{-1}$.

FIG. 15: Same as Figure 11 but for a resonance: $m_{\phi} / m_{\chi}=$ $10^{-3.84375}$ and $\alpha=10^{-2.625}$. 
the fact that there is much more space far away from the center of the galaxy, and because of the strong tidal forces near the center of the galaxy, which strongly affects halos which pass near the center of the galaxy.

For each halo we can estimate the velocity dispersion using the mass-velocity dispersion relation given in equation (34) and we can calculate the characteristic density $\rho_{s}$ and the characteristic length-scale $r_{s}$ from the values of $v_{\max }$ and $r_{\max }$ given for each subhalo, by the procedure outlined at the end of Sec. III B. For a given set of particle physics parameters $m_{\phi} / m_{\chi}$ and $\alpha$ this allows us to calculate the total boost to the DM annihilation cross section for each of the subhalos, and by using the assumptions about the particle physics given in Sec. III A we can also calculate the photon flux from DM annihilation coming from each subhalo, and the total gamma-ray flux coming from all of the subhalos combined.

We only cover some typical cases for the Sommerfeld enhancement here which suffices to show the general trend. We consider the particle physics parameters $m_{\phi} / m_{\chi}$ and $\alpha$ which give rise to the smallest Sommerfeld enhancements in Figures 10 and 11, i.e., $m_{\phi} / m_{\chi}=10^{-2}$ and $\alpha=0.001$. The top panel of Figure 10 shows the distribution of gamma-ray fluxes from DM annihilation for the subhalos, while the bottom panel shows the distribution of the total boost factors. As can be seen, the total boost factors are quite small: the typical boost factor is only about 30 with the highest boost factors being about 100. The total gamma-ray flux coming from subhalos is then also quite small in this case, as is shown in Figure 11, in which the total gamma-ray flux is shown for 1,000 random observers at a distance of $8.5 \mathrm{kpc}$ from the galactic center. At most observation locations this total flux is about $10^{-7} \mathrm{~cm}^{2} \mathrm{~s}^{-1}$, with the high-end tail not extending further than about half an order of magnitude from this. For comparison the dashed histogram in Figure 11 shows the same distribution of total gamma-ray flux for different random observers in the absence of any Sommerfeld enhancement. This confirms that the total gamma-ray flux with Sommerfeld enhancement is about an order of magnitude larger than without Sommerfeld enhancement.

Figures 12 and 13 show the same for a different extremum of the Sommerfeld enhancement: the case in which $m_{\phi} / m_{\chi}=10^{-5}$ and $\alpha=0.1$. As we saw before, in this case the Sommerfeld enhancement can reach very high values, and thus we see that the typical DM annihilation signal coming from DM subhalos is large: the typical flux for one subhalo is equal to the total flux in the scenario described in the previous paragraph. The total boost factors for most of the halos are very large, of the order of $10^{7}$, which gives rise to this very large photonflux. The total predicted flux in this case is many orders of magnitude larger than the total flux in the other extremum of the Sommerfeld enhancement and about seven orders of magnitude larger than the total gamma-ray flux in the absence of any Sommerfeld enhancement. Thus, the exact value of the parameters $m_{\phi} / m_{\chi}$ and $\alpha$, upon which the Sommerfeld enhancement depends, matters a great deal for the predicted gamma-ray flux.

Finally, we look at the expected signal coming from subhalos when the Sommerfeld enhancement is at a resonance, the same distribution as before are shown in Figures 14 and 15. Again we find large boost factors and large individual and total gamma-ray fluxes, although they are smaller than the values found for the $m_{\phi} / m_{\chi}=$ $10^{-5}$ and $\alpha=0.1$ parameter set. However, since these values are near a resonance in a region of parameter space around which the predicted signal looks more like that for the parameter set $m_{\phi} / m_{\chi}=10^{-2}$ and $\alpha=0.001$, this again shows that whether dark halos, i.e., subhalos without star formation, can be detected through DM annihilation products depends very sensitively on the particle physics parameters upon which the Sommerfeld enhancement depends.

In conclusion we can say that in a scenario in which there is Sommerfeld enhancement there is much more hope to detect dark subhalos than in more conventional scenarios, but that the details of the prediction depend strongly on the underlying particle physics.

\section{CONCLUSION}

The recently proposed Sommerfeld enhancement to the DM annihilation cross section forces us to reconsider statements made in the past about the expected signal of DM annihilation from cold substructures in the halo of the Galaxy. Since the Sommerfeld enhancement depends critically on the velocity dispersion of the substructure in question, as well as on the details of the particle physics model used to describe the attractive (or, possibly, repulsive) force that gives rise to the Sommerfeld enhancement, the contributions from the specific particle physics model and the details of the DM distribution in the substructure can no longer be as cleanly separated as they have been before. This means that in a scenario in which there is a significant Sommerfeld enhancement, previous claims of "particle physics independent" conclusions about the DM annihilation signal from substructure (e.g., $[39,65])$ need to be reconsidered in this new framework. This complicates the analysis but it opens up new possibilities for detecting DM annihilation and learning about the properties of the dark sector. As we showed above, the combination of the Sommerfeld enhancement and the substructure that is believed to exist down to small scales leads to very large boost factors because of the combined effect of the higher densities of small subhalos as well as the fact that they are kinematically colder than the larger halos. This leads to predictions for the DM annihilation gamma-ray flux that are much larger than was previously believed. As we showed in Sec. IV, in models in which there is a significant Sommerfeld enhancement at the level of the Galactic halo and its subhalos, the DM annihilation signal from the dwarf Spheroidals outshines the extragalactic background flux and the pre- 
dicted gamma-ray fluxes are so large that they should be detectable by Fermi. Given a detailed knowledge of the density structure and velocity dispersion profile of the dwarf Spheroidal galaxies, much can then be learned if we are able to detect the DM annihilation signal coming from these dSphs, by considering the relative signal and using the results of Sec. IV, or by using the detailed velocity dispersion profile of the dSphs [161].

We have focused on the consequences of the Sommerfeld enhancement for DM subhalos in a $\Lambda \mathrm{CDM}$ cosmology, using the latest results from high-resolution numerical $N$-body simulations to inform our assumptions about the abundance and properties of the substructure. Under these assumptions we were able to show that there are very large boosts to the DM annihilation cross section from the combined effect of substructure boosts and Sommerfeld enhancement in large parts of the parameter space consisting of the parameters of the attractive Yukawa type force between the DM particles which is responsible for the Sommerfeld enhancement. These regions of parameter space are in no way unrealistic. Indeed, in one of the most straightforward interpretations of the ATIC/PAMELA results, the sharp cut-off around $1 \mathrm{TeV}$ seen in the ATIC spectrum is interpreted as being a consequence of the fact that the dark matter mass is about $700 \mathrm{GeV}$. The absence of an excess in anti-protons similar to the excess in positrons seen by PAMELA can be explained by positing that the dark matter particles annihilate to an intermediate boson $\phi$, which is lighter than a proton (or even, lighter than the effective quark mass $\Lambda_{\mathrm{QCD}}$ ) in order to kinematically forbid the annihilation into protons [30]. This naturally leads to a $m_{\phi} / m_{\chi} \lesssim 10^{-3}$. The coupling constant of the new force in the dark sector is also generally $\alpha \gtrsim 10^{-2}[8,26]$. Thus, from Figure 5, we expect there to be a rather large boost to the DM annihilation cross section in these models. This leads to particularly large DM annihilation signals predicted for the dSphs (see Figure 8).

We have not discussed the substructure boost to the
DM annihilation signal from unresolved substructure, such as very small subhalos and DM tidal debris from disrupted subhalos, in the Galactic halo. This is, however, interesting in the light of the Sommerfeld enhancement, since it might lead to a different prediction about the relative magnitudes of the annihilation signal coming from the resolved Galactic halo and unresolved halo, as well from the resolved substructures in the Galactic halos. It is already clear that in the generic $1 / v$ Sommerfeld enhancement the main halo, with its typical velocity dispersion of $\sim 150 \mathrm{~km} \mathrm{~s}^{-1}$, will receive a much smaller enhancement than the dSphs, with their typical velocity dispersions of $10 \mathrm{~km} \mathrm{~s}^{-1}$. This, however, ignores the effects of saturation and resonances, which might be important in the specific particle physics model adopted for the Sommerfeld enhancement. Additionally, while the velocity dispersion of the Galactic halo might be of the order of $100 \mathrm{~km} \mathrm{~s}^{-1}$, the actual velocity dispersion relevant to the Sommerfeld enhancement might be much smaller for halo DM particles. If a large fraction of the DM in the Galactic halo is part of a cold stream - and various numerical simulations predict that large parts of the halo, if not all of the halo, are made up of such cold streams with typical velocity dispersions of the order of $1 \mathrm{~km} \mathrm{~s}^{-1}[59,60]$ - then, in addition to any boost caused by density enhancements in the stream [162], the boost from the Sommerfeld enhancement could also play a large role, as the relevant relative velocity of DM particles is set by the velocity dispersion of particles in the stream, and not by the velocity dispersion of the streams in the halo, which is what the $100 \mathrm{~km} \mathrm{~s}^{-1}$ Galactic halo velocity dispersion corresponds to in these scenarios. Thus, the expected DM annihilation signal from the main halo could be much larger than that naively expected for a halo with a velocity dispersion of $100 \mathrm{~km} \mathrm{~s}^{-1}$.

Acknowledgments: It is a pleasure to thank Ilias Cholis, Mulin Ding, David Hogg, and Neal Weiner for comments and assistance. This research was partially supported by NASA (grant NNX08AJ48G).
[1] E. Komatsu et al., Astrophys. J. Suppl. 180, 330 (2009).

[2] G. Jungman, M. Kamionkowski, and K. Griest, Phys. Rep. 267, 195 (1996).

[3] J. Silk and A. Stebbins, Astrophys. J. 411, 439 (1993).

[4] L. Bergström, J. Edsjö, P. Gondolo, and P. Ullio, Phys. Rev. D 59, 043506 (1999).

[5] J. Lavalle, Q. Yuan, D. Maurin, and X.-J. Bi, Astron. Astrophys. 479, 427 (2008).

[6] M. Kuhlen, J. Diemand, and P. Madau, Astrophys. J. 686, 262 (2008).

[7] S. Profumo, Phys. Rev. D 72, 103521 (2005).

[8] M. Lattanzi and J. Silk, arXiv:0812.0360 [astro-ph] (2008).

[9] O. Adriani et al., Nature 458, 607 (2009).

[10] J. Chang et al., Nature 456, 362 (2008).

[11] J. L. Fanselow, R. C. Hartman, R. H. Hildebrad, and P. Meyer, Astrophys. J. 158, 771 (1969).
[12] A. Buffington, C. D. Orth, and G. F. Smoot, Astrophys. J. 199, 669 (1975).

[13] D. Müller and K.-K. Tang, Astrophys. J. 312, 183 (1987).

[14] R. L. Golden et al., Astrophys. J. 436, 769 (1994).

[15] S. W. Barwick et al. (HEAT), Astrophys. J. 482, L191 (1997).

[16] J. J. Beatty et al., Phys. Rev. Lett. 93, 241102 (2004).

[17] M. Aguilar et al. (AMS-01), Phys. Lett. B 646, 145 (2007).

[18] G. L. Kane, L.-T. Wang, and T. T. Wang, Phys. Lett. B 536, 263 (2002).

[19] D. Hooper, J. E. Taylor, and J. Silk, Phys. Rev. D 69, 103509 (2004).

[20] I. Cholis, D. P. Finkbeiner, L. Goodenough, and N. Weiner, arXiv:0810.5344 [astro-ph] (2008).

[21] F. A. Aharonian, A. M. Atoyan, and H. J. Voelk, As- 
tron. Astrophys. 294, L41 (1995).

[22] D. Hooper, P. Blasi, and P. D. Serpico, J. Cosmol. Astropart. Phys. 1, 25 (2009).

[23] H. Yuksel, M. D. Kistler, and T. Stanev, arXiv:0810.2784 [astro-ph] (2008).

[24] S. Profumo, arXiv:0812.4457 [astro-ph] (2008).

[25] R. Harnik and G. D. Kribs, arXiv:0810.5557 [hep-ph] (2008).

[26] N. Arkani-Hamed, D. P. Finkbeiner, T. R. Slatyer, and N. Weiner, Phys. Rev. D 79, 015014 (2009).

[27] P. J. Fox and E. Poppitz, arXiv:0811.0399 [hep-ph] (2008).

[28] A. Ibarra and D. Tran, J. Cosmol. Astropart. Phys. 2, 21 (2009), 0811.1555.

[29] C.-R. Chen, M. M. Nojiri, F. Takahashi, and T. T. Yanagida, arXiv:0811.3357 [astro-ph] (2008).

[30] I. Cholis, G. Dobler, D. P. Finkbeiner, L. Goodenough, and N. Weiner, arXiv:0811.3641 [astro-ph] (2008).

[31] R. Allahverdi, B. Dutta, K. Richardson-McDaniel, and Y. Santoso, arXiv:0812.2196 [hep-ph] (2008).

[32] D. P. Finkbeiner, Astrophys. J. 614, 186 (2004).

[33] G. Dobler and D. P. Finkbeiner, Astrophys. J. 680, 1222 (2008).

[34] D. Hooper, D. P. Finkbeiner, and G. Dobler, Phys. Rev. D 76, 083012 (2007).

[35] A. W. Strong et al., Astron. Astrophys. 444, 495 (2005).

[36] F. A. Aharonian et al. (H.E.S.S.), Phys. Rev. Lett. 101, 261104 (2008).

[37] O. Adriani et al., Phys. Rev. Lett. 102, 051101 (2009).

[38] J. Diemand, M. Kuhlen, P. Madau, M. Zemp, B. Moore, D. Potter, and J. Stadel, Nature 454, 735 (2008), 0805.1244.

[39] V. Springel, S. D. M. White, C. S. Frenk, J. F. Navarro, A. Jenkins, M. Vogelsberger, J. Wang, A. Ludlow, and A. Helmi, Nature 456, 73 (2008).

[40] A. Sommerfeld, Annalen der Physik 403, 257 (1931).

[41] J. Hisano, S. Matsumoto, and M. M. Nojiri, Phys. Rev. D 67, 075014 (2003).

[42] J. Hisano, S. Matsumoto, and M. M. Nojiri, Phys. Rev. Lett. 92, 031303 (2004).

[43] J. Hisano, S. Matsumoto, M. M. Nojiri, and O. Saito, Phys. Rev. D 71, 063528 (2005).

[44] J. Hisano, S. Matsumoto, M. M. Nojiri, and O. Saito, Phys. Rev. D 71, 015007 (2005).

[45] J. Hisano, S. Matsumoto, O. Saito, and M. Senami, Phys. Rev. D 73, 055004 (2006).

[46] M. Cirelli, R. Franceschini, and A. Strumia, Nucl. Phys. B 800, 204 (2008).

[47] J. March-Russell, S. M. West, D. Cumberbatch, and D. Hooper, J. High. Energy. Phys. 7, 58 (2008).

[48] J. March-Russell and S. M. West, arXiv:0812.0559 [astro-ph] (2008).

[49] M. Kamionkowski and S. Profumo, Phys. Rev. Lett. 101, 261301 (2008).

[50] R. A. Ibata, G. Gilmore, and M. J. Irwin, Nature 370, 194 (1994).

[51] A. Helmi, S. D. M. White, P. T. de Zeeuw, and H. Zhao, Nature 402, 53 (1999).

[52] B. Willman et al., Astrophys. J. 626, L85 (2005).

[53] V. Belokurov et al., Astrophys. J. 654, 897 (2007).

[54] S. E. Koposov et al., Astrophys. J. 686, 279 (2008).

[55] S. Ghigna, B. Moore, F. Governato, G. Lake, T. Quinn, and J. Stadel, Mon. Not. Roy. Astron. Soc. 300, 146 (1998).
[56] K. V. Johnston, Astrophys. J. 495, 297 (1998).

[57] B. Moore, S. Ghigna, F. Governato, G. Lake, T. Quinn, J. Stadel, and P. Tozzi, Astrophys. J. 524, L19 (1999).

[58] A. Klypin, A. V. Kravtsov, O. Valenzuela, and F. Prada, Astrophys. J. 522, 82 (1999).

[59] A. Helmi and S. D. M. White, Mon. Not. Roy. Astron. Soc. 307, 495 (1999).

[60] A. Helmi, S. D. M. White, and V. Springel, Mon. Not. Roy. Astron. Soc. 339, 834 (2003).

[61] V. Springel, J. Wang, M. Vogelsberger, A. Ludlow, A. Jenkins, A. Helmi, J. F. Navarro, C. S. Frenk, and S. D. M. White, Mon. Not. Roy. Astron. Soc. 391, 1685 (2008).

[62] E. A. Baltz and L. Wai, Phys. Rev. D 70, 023512 (2004).

[63] S. M. Koushiappas, A. R. Zentner, and T. P. Walker, Phys. Rev. D 69, 043501 (2004).

[64] J. Diemand, M. Kuhlen, and P. Madau, Astrophys. J. 657, 262 (2007).

[65] L. E. Strigari, S. M. Koushiappas, J. S. Bullock, and M. Kaplinghat, Phys. Rev. D 75, 083526 (2007).

[66] L. Pieri, G. Bertone, and E. Branchini, Mon. Not. Roy. Astron. Soc. 384, 1627 (2008).

[67] L. E. Strigari, S. M. Koushiappas, J. S. Bullock, M. Kaplinghat, J. D. Simon, M. Geha, and B. Willman, Astrophys. J. 678, 614 (2008).

[68] M. Geha, B. Willman, J. D. Simon, L. E. Strigari, E. N. Kirby, D. R. Law, and J. Strader, 692, 1464 (2009).

[69] M. L. Mateo, Ann. Rev. Astron. Astrophys. 36, 435 (1998).

[70] J. D. Simon and M. Geha, Astrophys. J. 670, 313 (2007).

[71] J. S. Bullock, T. S. Kolatt, Y. Sigad, R. S. Somerville, A. V. Kravtsov, A. A. Klypin, J. R. Primack, and A. Dekel, Mon. Not. Roy. Astron. Soc. 321, 559 (2001).

[72] V. R. Eke, J. F. Navarro, and M. Steinmetz, Astrophys. J. 554, 114 (2001).

[73] M. G. Walker, M. Mateo, E. W. Olszewski, O. Y. Gnedin, X. Wang, B. Sen, and M. Woodroofe, Astrophys. J. 667, L53 (2007).

[74] J. Mardon, Y. Nomura, D. Stolarski, and J. Thaler, arXiv:0901.2926 [hep-ph] (2009).

[75] L. Bergström, P. Ullio, and J. H. Buckley, Astropart. Phys. 9, 137 (1998).

[76] N. Fornengo, L. Pieri, and S. Scopel, Phys. Rev. D 70, 103529 (2004).

[77] J. F. Navarro, C. S. Frenk, and S. D. M. White, Mon. Not. Roy. Astron. Soc. 275, 720 (1995).

[78] J. F. Navarro, C. S. Frenk, and S. D. M. White, Astrophys. J. 462, 563 (1996).

[79] J. F. Navarro, C. S. Frenk, and S. D. M. White, Astrophys. J. 490, 493 (1997).

[80] A. F. Neto, L. Gao, P. Bett, S. Cole, J. F. Navarro, C. S. Frenk, S. D. M. White, V. Springel, and A. Jenkins, Mon. Not. Roy. Astron. Soc. 381, 1450 (2007).

[81] L. Gao, J. F. Navarro, S. Cole, C. S. Frenk, S. D. M. White, V. Springel, A. Jenkins, and A. F. Neto, Mon. Not. Roy. Astron. Soc. 387, 536 (2008).

[82] S. Cole and C. Lacey, Mon. Not. Roy. Astron. Soc. 281, 716 (1996).

[83] G. Tormen, F. R. Bouchet, and S. D. M. White, Mon. Not. Roy. Astron. Soc. 286, 865 (1997).

[84] A. V. Kravtsov, A. A. Klypin, and A. M. Khokhlov, Astrophys. J. Suppl. 111, 73 (1997). 
[85] T. Fukushige and J. Makino, Astrophys. J. 477, L9 (1997).

[86] B. Moore, F. Governato, T. Quinn, J. Stadel, and G. Lake, Astrophys. J. 499, L5 (1998).

[87] B. Moore, T. Quinn, F. Governato, J. Stadel, and G. Lake, Mon. Not. Roy. Astron. Soc. 310, 1147 (1999).

[88] S. Ghigna, B. Moore, F. Governato, G. Lake, T. Quinn, and J. Stadel, Astrophys. J. 544, 616 (2000).

[89] A. Klypin, A. V. Kravtsov, J. S. Bullock, and J. R. Primack, Astrophys. J. 554, 903 (2001).

[90] T. Fukushige and J. Makino, Astrophys. J. 557, 533 (2001).

[91] J. Diemand, B. Moore, and J. Stadel, Mon. Not. Roy. Astron. Soc. 353, 624 (2004).

[92] A. V. Kravtsov, A. A. Klypin, J. S. Bullock, and J. R. Primack, Astrophys. J. 502, 48 (1998).

[93] C. Power, J. F. Navarro, A. Jenkins, C. S. Frenk, S. D. M. White, V. Springel, J. Stadel, and T. Quinn, Mon. Not. Roy. Astron. Soc. 338, 14 (2003).

[94] Y. P. Jing and Y. Suto, Astrophys. J. 529, L69 (2000).

[95] Y. P. Jing, Astrophys. J. 535, 30 (2000).

[96] J. Diemand, M. Zemp, B. Moore, J. Stadel, and C. M. Carollo, Mon. Not. Roy. Astron. Soc. 364, 665 (2005).

[97] S. R. Knollmann, C. Power, and A. Knebe, Mon. Not. Roy. Astron. Soc. 385, 545 (2008).

[98] J. Stadel, D. Potter, B. Moore, J. Diemand, P. Madau, M. Zemp, M. Kuhlen, and V. Quilis, arXiv:0808.2981 [astro-ph] (2008).

[99] J. F. Navarro, A. Ludlow, V. Springel, J. Wang, M. Vogelsberger, S. D. M. White, A. Jenkins, C. S. Frenk, and A. Helmi, arXiv:0810.1522 [astro-ph] (2008).

[100] J. F. Navarro, E. Hayashi, C. Power, A. R. Jenkins, C. S. Frenk, S. D. M. White, V. Springel, J. Stadel, and T. R. Quinn, Mon. Not. Roy. Astron. Soc. 349, 1039 (2004).

[101] D. Merritt, J. F. Navarro, A. Ludlow, and A. Jenkins, Astrophys. J. 624, L85 (2005).

[102] D. Merritt, A. W. Graham, B. Moore, J. Diemand, and B. Terzić, Astron. J. 132, 2685 (2006).

[103] A. W. Graham, D. Merritt, B. Moore, J. Diemand, and B. Terzić, Astron. J. 132, 2701 (2006).

[104] F. Stoehr, Mon. Not. Roy. Astron. Soc. 365, 147 (2006).

[105] J. Einasto, Trudy Inst. Astrofiz. Alma-Ata 5, 87 (1965).

[106] J. Einasto and U. Haud, Astron. Astrophys. 223, 89 (1989).

[107] P. Gondolo and J. Silk, Phys. Rev. Lett. 83, 1719 (1999).

[108] C. Calcáneo-Roldán and B. Moore, Phys. Rev. D 62, 123005 (2000).

[109] P. Gondolo, Phys. Lett. B 494, 181 (2000).

[110] C. Tyler, Phys. Rev. D 66, 023509 (2002).

[111] P. Ullio, L. Bergström, J. Edsjö, and C. Lacey, Phys. Rev. D 66, 123502 (2002).

[112] J. E. Taylor and J. Silk, Mon. Not. Roy. Astron. Soc. 339, 505 (2003).

[113] R. Aloisio, P. Blasi, and A. V. Olinto, Astrophys. J. 601, 47 (2004).

[114] E. A. Baltz et al., J. Cosmol. Astropart. Phys. 7, 13 (2008).

[115] G. Bertone, M. Cirelli, A. Strumia, and M. Taoso, J. Cosmol. Astropart. Phys. 3, 9 (2009), 0811.3744.

[116] L. Bergstrom, G. Bertone, T. Bringmann, J. Edsjo, and M. Taoso, arXiv:0812.3895 [astro-ph] (2008).

[117] V. R. Eke, S. Cole, and C. S. Frenk, Mon. Not. Roy. As- tron. Soc. 282, 263 (1996).

[118] G. L. Bryan and M. L. Norman, Astrophys. J. 495, 80 (1998).

[119] C. Schmid, D. J. Schwarz, and P. Widerin, Phys. Rev. D 59, 043517 (1999).

[120] S. Hofmann, D. J. Schwarz, and H. Stöcker, Phys. Rev. D 64, 083507 (2001).

[121] A. M. Green, S. Hofmann, and D. J. Schwarz, Mon. Not. Roy. Astron. Soc. 353, L23 (2004).

[122] A. Loeb and M. Zaldarriaga, Phys. Rev. D 71, 103520 (2005).

[123] S. Profumo, K. Sigurdson, and M. Kamionkowski, Phys. Rev. Lett. 97, 031301 (2006).

[124] J. Diemand, M. Kuhlen, and P. Madau, Astrophys. J. 649, 1 (2006).

[125] A. Helmi, S. D. White, and V. Springel, Phys. Rev. D 66, $063502(2002)$.

[126] L. Gao, S. D. M. White, A. Jenkins, F. Stoehr, and V. Springel, Mon. Not. Roy. Astron. Soc. 355, 819 (2004)

[127] A. R. Zentner and J. S. Bullock, Astrophys. J. 598, 49 (2003).

[128] J. Diemand, M. Kuhlen, and P. Madau, Astrophys. J. 667, 859 (2007).

[129] L. D. Shaw, J. Weller, J. P. Ostriker, and P. Bode, Astrophys. J. 659, 1082 (2007).

[130] A. V. Macciò, A. A. Dutton, F. C. van den Bosch, B. Moore, D. Potter, and J. Stadel, Mon. Not. Roy. Astron. Soc. 378, 55 (2007).

[131] M. Kuhlen, L. E. Strigari, A. R. Zentner, J. S. Bullock, and J. R. Primack, Mon. Not. Roy. Astron. Soc. 357, 387 (2005).

[132] J. Peñarrubia, J. F. Navarro, and A. W. McConnachie, Astrophys. J. 673, 226 (2008).

[133] E. Hayashi, J. F. Navarro, C. Power, A. Jenkins, C. S. Frenk, S. D. M. White, V. Springel, J. Stadel, and T. R. Quinn, Mon. Not. Roy. Astron. Soc. 355, 794 (2004).

[134] J. S. Bullock and K. V. Johnston, Astrophys. J. 635, 931 (2005).

[135] S. Kazantzidis, L. Mayer, C. Mastropietro, J. Diemand, J. Stadel, and B. Moore, Astrophys. J. 608, 663 (2004).

[136] R. Mandelbaum, U. Seljak, and C. M. Hirata, J. Cosmol. Astropart. Phys. 8, 6 (2008).

[137] J. Binney and S. Tremaine, Galactic Dynamics: Second Edition (Princeton University Press, 2008).

[138] A. E. Evrard and J. P. Henry, Astrophys. J. 383, 95 (1991).

[139] M. M. Crone and M. J. Geller, Astron. J. 110, 21 (1995).

[140] A. E. Evrard, C. A. Metzler, and J. F. Navarro, Astrophys. J. 469, 494 (1996).

[141] J. Peñarrubia, A. W. McConnachie, and J. F. Navarro, Astrophys. J. 672, 904 (2008).

[142] E. A. Baltz, C. Briot, P. Salati, R. Taillet, and J. Silk, Phys. Rev. D 61, 023514 (2000).

[143] N. W. Evans, F. Ferrer, and S. Sarkar, Phys. Rev. D 69, 123501 (2004).

[144] S. Peirani, R. Mohayaee, and J. A. de Freitas Pacheco, Phys. Rev. D 70, 043503 (2004).

[145] D. Hooper, F. Ferrer, C. Boehm, J. Silk, J. Paul, N. W. Evans, and M. Casse, Phys. Rev. Lett. 93, 161302 (2004).

[146] L. Bergström and D. Hooper, Phys. Rev. D 73, 063510 (2006).

[147] S. Profumo and M. Kamionkowski, J. Cosmol. As- 
tropart. Phys. 3, 3 (2006).

[148] S. Colafrancesco, S. Profumo, and P. Ullio, Phys. Rev. D 75, 023513 (2007).

[149] M. A. Sánchez-Conde, F. Prada, E. L. Łokas, M. E. Gómez, R. Wojtak, and M. Moles, Phys. Rev. D 76, 123509 (2007).

[150] L. Pieri, A. Pizzella, E. M. Corsini, E. Dalla Bonta', and F. Bertola, Astron. Astrophys. 496, 351 (2009), 0812.1494 .

[151] L. Pieri, M. Lattanzi, and J. Silk, arXiv:0902.4330 [astro-ph] (2009).

[152] M. Wood et al., Astrophys. J. 678, 594 (2008).

[153] J. Albert et al., Astrophys. J. 679, 428 (2008).

[154] F. Aharonian et al., Astropart. Phys. 29, 55 (2008).
[155] D. D. Driscoll et al., Phys. Rev. D 78, 087101 (2008).

[156] F. Aharonian et al., Astrophys. J. 691, 175 (2009).

[157] E. Aliu and others (MAGIC), arXiv:0810.3561 [astroph] (2008).

[158] M. G. Walker, M. Mateo, E. W. Olszewski, B. Sen, and M. Woodroofe, Astron. J. 137, 3109 (2009).

[159] A. W. Strong, I. V. Moskalenko, and O. Reimer, Astrophys. J. 613, 962 (2004).

[160] P. Sreekumar et al., Astrophys. J. 494, 523 (1998).

[161] B. Robertson and A. Zentner, arXiv:0902.0362 [astroph] (2009).

[162] N. Afshordi, R. Mohayaee, and E. Bertschinger, arXiv: 0811.1582 [astro-ph] (2008). 